\title{
Translating genomics into practice for real-time surveillance and response to carbapenemase-producing Enterobacteriaceae: evidence from a complex multi- institutional KPC outbreak
}

\author{
Jason C Kwong ${ }^{1,2,3}$, Courtney Lane ${ }^{4,5}$, Finn Romanes ${ }^{5}$, Anders Gonçalves da Silva ${ }^{1,4}$, Marion Easton ${ }^{5}$, Katie \\ Cronin ${ }^{6}$, Mary Jo Waters ${ }^{6}$, Takehiro Tomita ${ }^{4}$, Kerrie Stevens ${ }^{4}$, Mark B Schultz ${ }^{1,4}$, Sarah L Baines ${ }^{1,2}$, \\ Norelle L Sherry ${ }^{1,2,3}$, Glen Carter ${ }^{1,2}$, Andre Mu ${ }^{1,2}$, Michelle Sait ${ }^{4}$, Susan A Ballard ${ }^{1,4}$, Torsten Seemann ${ }^{1,7}$, \\ Timothy P Stinear ${ }^{1,2}$, Benjamin P Howden Corresp. 1, 2, 3,4 \\ 1 Doherty Applied Microbial Genomics, The University of Melbourne at The Peter Doherty Institute for Infection and Immunity, Melbourne, Australia \\ 2 Department of Microbiology and Immunology, The University of Melbourne at The Peter Doherty Institute for Infection and Immunity, Melbourne, \\ Australia \\ 3 Department of Infectious Diseases, Austin Health, Heidelberg, Australia \\ 4 Microbiological Diagnostic Unit Public Health Laboratory, The University of Melbourne at The Peter Doherty Institute for Infection and Immunity, \\ Melbourne, Australia \\ 5 Health Protection Branch, Department of Health and Human Services, Victoria State Government, Melbourne, Australia \\ 6 Department of Microbiology, St Vincent's Hospital Melbourne, Fitzroy, Australia \\ 7 Melbourne Bioinformatics, The University of Melbourne, Carlton, Australia \\ Corresponding Author: Benjamin P Howden \\ Email address: bhowden@unimelb.edu.au
}

Background. Until recently, KPC-producing Enterobacteriaceae were rarely identified in Australia. Following an increase in the number of incident cases across the state of Victoria, we undertook a realtime combined genomic and epidemiological investigation. The scope of this study included identifying risk factors and routes of transmission, and investigating the utility of genomics to enhance traditional field epidemiology for informing management of established widespread outbreaks.

Methods. All KPC-producing Enterobacteriaceae isolates referred to the state reference laboratory from 2012 onwards were included. Whole-genome sequencing (WGS) was performed in parallel with a detailed descriptive epidemiological investigation of each case, using Illumina sequencing on each isolate. This was complemented with PacBio long-read sequencing on selected isolates to establish high-quality reference sequences and interrogate characteristics of KPC-encoding plasmids.

Results. Initial investigations indicated the outbreak was widespread, with 86 KPC-producing Enterobacteriaceae isolates (K. pneumoniae 92\%) identified from 35 different locations across metropolitan and rural Victoria between 2012-2015. Initial combined analyses of the epidemiological and genomic data resolved the outbreak into distinct nosocomial transmission networks, and identified healthcare facilities at the epicentre of KPC transmission. New cases were assigned to transmission networks in real-time, allowing focussed infection control efforts. PacBio sequencing confirmed a secondary transmission network arising from inter-species plasmid transmission. Insights from Bayesian transmission inference and analyses of within-host diversity informed the development of state-wide public health and infection control guidelines, including interventions such as an intensive approach to screening contacts following new case detection to minimise unrecognised colonisation.

Conclusions. A real-time combined epidemiological and genomic investigation proved critical to 
identifying and defining multiple transmission networks of KPC Enterobacteriaceae, while data from either investigation alone were inconclusive. The investigation was fundamental to informing infection control measures in real-time and the development of state-wide public health guidelines on carbapenemase producing Enterobacteriaceae surveillance and management. 
1 Full Title: Translating genomics into practice for real-time surveillance and response to

2 carbapenemase-producing Enterobacteriaceae: evidence from a complex multi-institutional

3 KPC outbreak

5 Authors: Jason C Kwong 1,2,3, Courtney Lane ${ }^{4,5}$, Finn Romanes ${ }^{5}$, Anders Gonçalves da Silva ${ }^{1,4}$,

6 Marion Easton ${ }^{5}$, Katie Cronin ${ }^{6}$, Mary Jo Waters ${ }^{6}$, Takehiro Tomita ${ }^{4}$, Kerrie Stevens ${ }^{4}$, Mark B

7 Schultz ${ }^{1,4}$, Sarah L Baines ${ }^{1,2}$, Norelle L Sherry ${ }^{1,2,3}$, Glen Carter ${ }^{1,2}$, Andre Mu ${ }^{1,2}$, Michelle Sait

8 4, Susan A Ballard ${ }^{1,4}$, Torsten Seemann ${ }^{1,7}$, Timothy P Stinear ${ }^{1,2}$, Benjamin P Howden $1,2,3,4$

${ }^{1}$ Doherty Applied Microbial Genomics, and ${ }^{2}$ Department of Microbiology and Immunology, The

11 University of Melbourne at The Peter Doherty Institute for Infection \& Immunity, Melbourne,

12 Australia

$13{ }^{3}$ Department of Infectious Diseases, Austin Health, Heidelberg, Australia

$14{ }^{4}$ Microbiological Diagnostic Unit Public Health Laboratory, The University of Melbourne at The

15 Peter Doherty Institute for Infection \& Immunity, Melbourne, Australia

165 Health Protection Branch, Department of Health and Human Services, Victoria State

17 Government, Melbourne, Australia

$18{ }^{6}$ Department of Microbiology, St Vincent's Hospital Melbourne, Fitzroy, Australia

$19{ }^{7}$ Melbourne Bioinformatics, The University of Melbourne, Carlton, Australia

21 Correspondence:

22 Prof Benjamin P Howden

23 Microbiological Diagnostic Unit Public Health Laboratory 
24 Doherty Institute for Infection \& Immunity

25792 Elizabeth Street

26 Melbourne, Victoria

27 Australia 3000

28 Email: bhowden@unimelb.edu.au

29 Phone: +61383445701 


\section{ABSTRACT}

Background. Until recently, KPC-producing Enterobacteriaceae were rarely identified in Australia. Following an increase in the number of incident cases across the state of Victoria, we undertook a real-time combined genomic and epidemiological investigation. The scope of this study included identifying risk factors and routes of transmission, and investigating the utility of genomics to enhance traditional field epidemiology for informing management of established widespread outbreaks.

Methods. All KPC-producing Enterobacteriaceae isolates referred to the state reference laboratory from 2012 onwards were included. Whole-genome sequencing (WGS) was performed in parallel with a detailed descriptive epidemiological investigation of each case, using Illumina sequencing on each isolate. This was complemented with PacBio long-read sequencing on selected isolates to establish high-quality reference sequences and interrogate characteristics of KPC-encoding plasmids.

Results. Initial investigations indicated the outbreak was widespread, with 86 KPC-producing Enterobacteriaceae isolates (K. pneumoniae 92\%) identified from 35 different locations across metropolitan and rural Victoria between 2012-2015. Initial combined analyses of the epidemiological and genomic data resolved the outbreak into distinct nosocomial transmission networks, and identified healthcare facilities at the epicentre of KPC transmission. New cases were assigned to transmission networks in real-time, allowing focused infection control efforts. PacBio sequencing confirmed a secondary transmission network arising from inter-species plasmid transmission. Insights from Bayesian transmission inference and analyses of within-host diversity informed the development of state-wide public health and infection control guidelines, including 
52 interventions such as an intensive approach to screening contacts following new case detection to

53 minimise unrecognised colonisation.

54 Conclusions. A real-time combined epidemiological and genomic investigation proved critical to 55 identifying and defining multiple transmission networks of KPC Enterobacteriaceae, while data

56 from either investigation alone were inconclusive. The investigation was fundamental to informing

57 infection control measures in real-time and the development of state-wide public health guidelines

58 on carbapenemase producing Enterobacteriaceae surveillance and management. 
59

60

61

62

63

64

65

66

67

68

69

70

71

72

73

74

75

76

77

78

79

80

81

\section{INTRODUCTION}

Carbapenemase-producing Enterobacteriaceae (CPE) are among the most urgent antimicrobial resistance threats worldwide (Centers for Disease Control and Prevention 2013). In addition to producing the carbapenemase, capable of inactivating almost all beta-lactam antibiotics, including penicillins, cephalosporins and carbapenems, these organisms frequently harbour multiple other antibiotic resistance genes and mutations (Queenan \& Bush 2007). Few therapeutic options exist, and the available options are often limited by tolerability and efficacy (Falagas et al. 2014; Tzouvelekis et al. 2012).

In Australia, carbapenemases have been rarely identified in Enterobacteriaceae, apart from the weakly carbapenem-hydrolysing $b l a_{\mathrm{IMP}-4}$ which has established low level endemicity (Australian Commission on Safety and Quality in Health Care 2013; Australian Group on Antimicrobial Resistance 2014). Although small outbreaks and limited patient-to-patient transmission of CPE in Australia have been described (Chang et al. 2015; Espedido et al. 2013; Kotsanas et al. 2013; Peleg et al. 2005; Tai et al. 2015), the majority of patients identified with CPE are thought to have acquired these organisms during international travel to endemic countries (Chua et al. 2014; Fernando et al. 2010; Poirel et al. 2010; Sidjabat et al. 2011; Sidjabat et al. 2013). Limited transmission in the community has also been reported (Blyth et al. 2014).

Klebsiella pneumoniae carbapenemases (KPCs) were first identified in the United States of America in 1996, but have since spread worldwide to be endemic in several countries including the USA, Israel, Italy, Greece, Brazil and China (Munoz-Price et al. 2013). Several gene variants have been reported from the KPC family, though the most commonly reported alleles are KPC-2 
82 and KPC-3, that differ by a single amino acid substitution (Tzouvelekis et al. 2012). The first

83 reported KPC-producing organism (KPC-2) in Australia was isolated in 2010 from a patient who

84 had returned to Sydney, New South Wales, after being hospitalised in Greece (Coatsworth et al. 85 2012). In 2012, KPC-2 was first identified in the state of Victoria in a patient who had been 86 repatriated to a metropolitan hospital also after a prolonged admission in a Greek hospital (Chua 87 et al. 2014).

Whole-genome sequencing (WGS) has emerged as a powerful tool for bacterial strain typing and outbreak investigation (Kwong et al. 2015), and has been used in public health to assess transmission of Listeria monocytogenes and other foodborne pathogens at jurisdictional, national and international levels (Kwong et al. 2016). It has also been used at a single institution level to investigate small-to-medium sized outbreaks of KPC-producing Enterobacteriaceae (Jiang et al. 2015; Kanamori et al. 2017; Marsh et al. 2015; Mathers et al. 2015; Ruppe et al. 2017; Snitkin et al. 2012; Weterings et al. 2015). While the details of who infected whom can be traced among a small group of individuals in conjunction with a detailed epidemiological investigation, the transmission dynamics of larger established outbreaks across multiple institutions are more difficult to resolve (Gilchrist et al. 2015).

Due to the increasing incidence of KPC-producing Enterobacteriaceae during 2012-2014 from multiple sources across the state of Victoria (approximate population 6 million), we undertook an outbreak investigation employing WGS in parallel with traditional outbreak epidemiology. At the time this combined genomics and epidemiological investigation was initiated in June 2014, half of the 30 cases reported were from two metropolitan hospitals that each reported prior internal 
105 outbreaks of KPC-producing K. pneumoniae - one in 2012 and the other in early 2014. However, 106 no clear epidemiological links had been identified between the two hospitals. Additionally, the 107 remaining cases not identified through those two hospitals comprised patients in seven other 108 healthcare facilities and in the community. Although all but one of the isolates were ST258 by 109 multilocus sequence typing, if and where transmission of KPC-producing K. pneumoniae was 110 occurring was unknown. Here we report the utility of advanced genomic approaches including 111 sampling for within-host clonal diversity, Bayesian transmission modelling, and plasmid genome 112 reconstruction to assess the transmission dynamics of the outbreak to address these questions and 113 inform infection control management in real-time during the course of the outbreak. 
114 MATERIALS AND METHODS

115 An overview of the workflow and methods used is shown in Figure 1.

116

117 Laboratory and genomic methods

118 Isolate selection and antimicrobial susceptibility assays. From 2012, following the first KPC

119 isolate in the state, all Victorian diagnostic microbiology laboratories were asked to refer suspected

120 carbapenemase-producing isolates to the Microbiological Diagnostic Unit Public Health

121 Laboratory (MDU PHL) for further testing. Suspected isolates were defined as Enterobacteriaceae

122 with a meropenem minimum inhibitory concentration (MIC) $\geq 0.5 \mathrm{mg} / \mathrm{L}$ or zone diameter (ZD)

$123 \leq 23 \mathrm{~mm}$, or an imipenem $\mathrm{MIC} \geq 2 \mathrm{mg} / \mathrm{L}$ or $\mathrm{ZD} \leq 23 \mathrm{~mm}$. Isolates with a positive colorimetric (e.g.

124 Rapidec $\AA$ CarbaNP (Nordmann et al. 2012)) or molecular (e.g. Cepheid GeneXpert® (Tenover et

125 al. 2013)) test were also requested. Duplicate KPC-producing Enterobacteriaceae isolates of the

126 same species from a patient during a single hospital admission were usually excluded by referring

127 laboratories. However, for some patients, multiple isolates were referred to MDU PHL if they

128 originated from different laboratories, and were subsequently included in the analyses.

130 Species identification was confirmed using matrix-assisted laser desorption/ionisation time-offlight (MALDI-TOF) mass spectrometry (VITEK $®$ MS, bioMérieux, France) with routine 132 susceptibilities tested on selected isolates using VITEK ${ }^{2} 2$ Compact (AST-N246 cards, bioMérieux, France). All suspected CPE isolates meeting the above criteria underwent testing for carbapenemase genes using polymerase chain reaction (PCR) (Supplementary Table S1). Isolates confirmed as KPC-producing organisms underwent WGS and were included in the genomic analyses, with the respective patients included in the epidemiological investigation. Isolates 
137 obtained prior to 2014 were sequenced retrospectively, while WGS was performed prospectively

138 during the outbreak on isolates collected from 2014 onwards.

139

140 Multiple colony sampling. To understand the genomic diversity present within a single individual

141 and assess the influence on reconstructing transmission networks, primary faecal specimens were

142 obtained from one patient. After overnight culture on Brilliance ${ }^{\mathrm{TM}}$ CRE (Thermo Fisher, Waltham,

143 Massachusetts, US) selective media, 10-15 colonies, including any that differed in colony

144 morphology, were selected from the plates for DNA extraction and WGS. Multiple colony

145 sampling and sequencing for other patient samples was not performed due to cost limitations.

147 DNA extraction and whole genome sequencing. Bacterial cultures were purified for DNA

148 extraction by two successive single colony selections after streaking onto horse blood agar

149 incubated overnight at $37^{\circ} \mathrm{C}$. DNA was extracted from a liquid suspension of the purified cultures

150 using the QIAmp DNA Mini Kit (Qiagen, Hilden, Germany) or the JANUS Chemagic Workstation

151 with the Chemagic Viral DNA/RNA kit (CMG-1033; PerkinElmer, Waltham, Massachusetts, US).

152

153 WGS was performed on the Illumina MiSeq or NextSeq platforms using Nextera XT libraries and

154 protocols (Illumina, San Diego, California, US) with a minimum average quality score of 30 and

155 a target sequencing depth of $\geq 50 \mathrm{x}$ as previously described (Carter et al. 2016). Isolates not meeting

156 these metrics were resequenced. Raw sequence data has been uploaded to the Sequence Read 157 Archive under BioProject PRJNA397262. 
159 Single molecule real-time (SMRT) sequencing of one Klebsiella pneumoniae and one Citrobacter

160 farmeri isolate was also performed on the PacBio RS II platform (Pacific Biosciences, Menlo Park,

161 California, US) using previously reported methods (Carter et al. 2016). In brief, genomic DNA

162 was extracted using a GenElute ${ }^{\mathrm{TM}}$ Bacterial Genomic DNA kit (Sigma-Aldrich, St. Louis,

163 Missouri, USA) and SMRTbell libraries were prepared using manufacturer protocols (Pacific

164 Biosciences, Menlo Park, California). Libraries underwent an additional size-selection step using 165 a 20-kb template BluePippin size-selection protocol (Sage Science, Beverly, Massachusetts, 166 USA).

167

Bioinformatic analyses. Illumina raw sequencing reads were trimmed to clip Nextera adapters and low-quality sequence (Phred score <10) using Trimmomatic v0.36 (Bolger et al. 2014). The trimmed reads were assembled de novo with the SPAdes v3.7.1 assembler (Bankevich et al. 2012) and auto-annotated with Prokka v1.12-beta (Seemann 2014). The SMRT analysis portal v2.3.0.140936 (Pacific Biosciences) was used for isolates sequenced on the PacBio RS II, with raw sequence reads assembled de novo using the HGAP3 protocol, and error correction and polishing with Quiver v1. Further error correction was performed by mapping Illumina short reads v3.2 (https://github.com/tseemann/snippy). The completed genome assemblies have been uploaded to GenBank under BioProject PRJNA397262.

From the annotated assemblies, the multi-locus sequence type (MLST) was determined in silico as was the presence or absence of antimicrobial resistance ("resistome") and other genes (pan- 
182 (https://github.com/tseemann/abricate) (https://github.com/sanger-pathogens/Roary) (Page et al.

183 2015). The pan-genome data was imported into FriPan (https://github.com/drpowell/FriPan) for

184 web-browser visualisation using a Python script (https://github.com/kwongj/roary2fripan), and

185 annotated genomes were visualised in Geneious v7.1.5 (http://www.geneious.com/).

186

187 Sequencing reads were also aligned to a reference genome to produce a reference-based whole188 genome alignment including single nucleotide polymorphism (SNP), invariant and missing sites 189 (https://github.com/tseemann/snippy). This alignment was then trimmed to exclude plasmid sites 190 (https://github.com/kwongj/trim-aln). Putative regions of recombination were predicted using ClonalFrameML v1.0 (Didelot \& Wilson 2015), and masked in the alignment 192 (https://github.com/kwongj/cfml-maskrc). Core genome SNP sites were extracted from the recombination-filtered alignment (Page et al. 2016) and a maximum likelihood phylogenetic tree inferred from the resulting SNP alignment in RAxML v8.2.4 (Stamatakis 2014), using a General Time Reversible model of nucleotide substitution with a $\Gamma$ model of rate heterogeneity and four rate categories, with 1000 bootstrap replicates to determine branch support. Hierarchical Bayesian Analysis of Population Structure (hierBAPS) (Cheng et al. 2013) was used to provide further support for identifying phylogenetic clades, with clustering performed using 8 levels in the hierarchy (L) and the prior maximum number of clusters (maxK) set at 10. Publicly available sequencing data was retrieved from the National Center for Biotechnology Information (NCBI)

Sequence Read Archive and GenBank for comparison with local genomes (Supplementary Tables S2 and S3). Three reference genomes were used. The initial genomic analysis was performed using KPNIH24 (GenBank accession CP008797), with PacBio sequences of internal isolates $(K$. 
AUSMDU00008141, GenBank accession SAMN07452765) used for subsequent analyses to minimise inaccuracies and biases from using distant genomes and draft genome assemblies as references (Supplementary Figure S1).

Genomic context of the bla $a_{\mathrm{KPC}}$ gene. The genomic context of the bla $a_{\mathrm{KPC}}$ gene was investigated using a custom Python script (https://github.com/kwongj/contig-puller) to extract and align de novo assembled contigs carrying the gene. The flanking $T n 4401$ transposon regions were compared to previously described isoforms (Chen et al. 2012; Kitchel et al. 2009; Naas et al. 2012; Naas et al. 2008), using BLAST+ (Camacho et al. 2009). Plasmid replicons were identified in genome assemblies using BLAST+ against the PlasmidFinder database (Carattoli et al. 2014). Plasmids were presumed to carry KPC if $b a_{\mathrm{KPC}}$ was identified on the same contiguous sequence of DNA as the plasmid replicon.

Bayesian evolutionary analyses and transmission modelling. Bayesian evolutionary analyses were also conducted to ascertain if the genomic signal could estimate the date of emergence of any outbreak clusters, and if any additional data could help inform where and when transmission events were taking place. A recombination-filtered chromosomal alignment was obtained as described above. The subsequent alignment was used as input into BEAST2 v2.4.3 (Bouckaert et al. 2014) with collection dates entered for each isolate. The relationship between root-to-tip distance and date of isolation was assessed in TempEst v1.5 (Rambaut et al. 2016). We fitted a model with a relaxed log-normal clock (Drummond et al. 2006) to the alignment to account for inter-clade variation, assuming a HKY model for nucleotide substitution with $\Gamma$ distributed among site rate variation (Hasegawa et al. 1985), and used a constant population size coalescent prior on the 
228 genealogy. Nodes were selected for logging the likely time of the most recent common ancestor

229 (MRCA) for major clades identified in the maximum likelihood tree. We used eight Markov chain

230 Monte Carlo (MCMC) runs of 100 million states, with sampling every 5000 states, and a burn-in

231 of 50 percent. The posterior samples from each chain were checked in Tracer v1.6

232 (http://tree.bio.ed.ac.uk/software/tracer/) for convergence, and then grouped into a single chain.

233 The posterior samples for the dates of the nodes of interest were annotated on a maximum clade

234 credibility tree and exported.

235

236 The maximum clade credibility tree was used as input into TransPhylo v1.0 (Didelot et al. 2017)

237 to reconstruct the transmission chain. We based our prior for generation time (the time from

238 primary infection of an individual to any subsequent secondary infection) on a previous study on

239 the duration of carriage of KPC-producing Enterobacteriaceae (Zimmerman et al. 2013), assuming

240 detection of KPC as an indicator of likelihood for onwards transmission. We therefore used a

241 Gamma distribution with shape parameter 1.2 and scale 1.0 for the generation time prior, with a

242 distribution mean of 1.2 years (438 days), standard deviation 1.096 years (400 days), and mode

2430.2 years (73 days). The MCMC was run for 100000 iterations, with transmissions inferred from

244 a consensus transmission tree. 
246 Outbreak genomic investigation. An initial retrospective analysis using the bioinformatic methods

247 described above was performed in June 2014 on 41 KPC-producing K. pneumoniae isolates that

248 had undergone WGS, including de novo genome assembly, in silico multi-locus sequence typing

249 (MLST), resistance gene detection and phylogenetic analysis. Subsequently, a customised, in-

250 house pipeline was developed to streamline and automate handling of sequence data for ongoing

251 assessment and analysis (https://github.com/tseemann/nullarbor). A summary report was

252 generated for each set of isolates run through Nullarbor including quality control metrics, MLST,

253 resistome and pan-genome comparison, and a maximum likelihood phylogeny inferred from the

254 core SNP alignment (Price et al. 2010).

255

256 From this initial report, clonal complex (CC) 258 isolates underwent further analysis using a

257 PacBio-assembled reference genome of a local isolate for a higher resolution core genome SNP

258 alignment and phylogenetic comparison. As additional KPC-positive isolates were identified over

259 time, the Nullarbor pipeline was repeated in an iterative process to establish the genomic

260 relationship of new isolates to existing isolates, and where new CC258 isolates were identified,

261 the CC258-specific analysis was also repeated with the new isolates. These analyses were

262 interpreted together with epidemiological data in real-time as new cases emerged (see below -

263 "Outbreak reporting and oversight"). Additional detailed analyses, including recombination

264 filtering and Bayesian temporal analyses, were performed ad hoc to gain an overall understanding

265 of the outbreak, and although were not used to directly inform infection control of individual cases,

266 were used to inform overall outbreak management and guideline development. 


\section{Epidemiological methods}

269 Following the results of the initial genomic analysis and concern regarding local transmission, the

270

271

272

273

274

275

276

277

278

279

280

281

282

283

284

285

286

287

288

289

290 ongoing genomic investigation was accompanied by collection of detailed epidemiological data, retrospectively for cases detected prior to 2014 and in real-time from 2014.

Data Collection. All patients from whom KPC-producing Enterobacteriaceae were identified from the first isolate in 2012 to 31 December 2015 were included in the epidemiological investigation. A case report form was developed to collect detailed epidemiological data, especially regarding patient hospitalisation details. Patients, or their next of kin, were interviewed by phone to ascertain demographic information on age, sex, country of birth, and risk factors for CPE such as hospitalisation and medical procedures, overseas travel, and comorbidities. Where a patient was in a healthcare facility at the time of specimen collection, or reported hospitalisation in the 12 months prior to initial $\mathrm{CPE}$ identification, medical records from each hospitalisation were examined to obtain data on specimen collection, clinical details of infection or colonisation, procedures and patient movements both before and after CPE identification, where available. Treating doctors, general practitioners and infection control personnel were contacted to obtain additional and/or missing information. Patient hospital admission data were collated and used to identify putative transmission networks of patients linked by proximity in time and space.

Once identified with KPC, patients were assumed to be colonised indefinitely, thus subsequent KPC isolates were deemed to constitute the same patient episode and epidemiological data were not re-collected. KPC-producing Enterobacteriaceae isolated from normally sterile sample sites e.g. blood, cerebrospinal fluid, pleural fluid, were considered to represent "infections". Isolates 
291 obtained from non-sterile sites (e.g. urine, wound swab, aspirate from intra-abdominal collection)

292 where clinical evidence of infection was present and the patient was treated with antibiotics with

293 activity against KPC-producing organisms, or where the treating clinician identified infection but

294 elected to palliate the patient, were also considered to represent "infections". Other isolates were

295 considered to represent "colonisations", unless no epidemiological data were available

296 ("unknown”).

297

298 Infection control investigation

299 For patients where local transmission of KPC-producing Enterobacteriaceae was suspected, 300 interviews were conducted with infection control practitioners (ICPs) at the facility with putative 301 transmission. Data were collected on environmental and contact screening activities, patient follow 302 up and patient management alerts, isolation and collection of subsequent screening specimens.

\section{Outbreak reporting and oversight}

Data from both genomic and epidemiological investigations were analysed together in real-time, 306 and reported back to the Victorian Department of Health and Human Services. Where transmission events were recognised, the healthcare institutions involved were also informed. An incident management team was established through the Department of Health and Human Services to oversee the investigation and management of the outbreak.

\section{Ethics approval and consent to participate}

312 Data were collected as part of an outbreak investigation through the Victorian Department of 313 Health and Human Services under the Public Health and Wellbeing Act 2008 
314 (https://www2.health.vic.gov.au/about/legislation/public-health-and-wellbeing-act). No consent

315 was required for the project.

316

317 Availability of data and materials

318 The raw Illumina short-read sequencing data and completed PacBio genome assemblies supporting

319 the conclusions of this article are available in the National Center for Biotechnology Information

320 database under BioProject PRJNA397262. In-house scripts used in the bioinformatics analyses are

321 available at https://github.com/tseemann and https://github.com/kwongj. 


\section{RESULTS}

323 A total of 86 KPC-producing Enterobacteriaceae were referred to MDU PHL between 2012-2015

324 from 69 patients in 26 different healthcare facilities, six general practices and three aged care

325 facilities across both metropolitan and rural Victoria (Figure 2). Specimen source was reported for

32685 of the isolates, with most specimens being urine $(n=42 ; 49 \%)$, faeces or rectal swabs $(n=20$;

$32723 \%)$, or blood cultures $(\mathrm{n}=10 ; 12 \%)$ (Supplementary Table S4). Almost all isolates were KPC-

3282 producing Enterobacteriaceae, apart from two KPC-3 producing K. pneumoniae isolates from

329 one patient. Overall, 79 of the isolates were K. pneumoniae, five were Citrobacter farmeri, with

330 single isolates of Klebsiella oxytoca and Citrobacter freundii.

\section{Initial genomic analysis}

333 In the initial retrospective genomic analysis of isolates collected prior to June 2014, 40/41 (98\%)

334 K. pneumoniae isolates collected from 29 patients were clonal complex (CC) 258 by in silico

335 MLST, with all of these belonging to clade 1 of sequence type (ST) $258 \mathrm{~K}$. pneumoniae described

336 by Deleo et al. (Deleo et al. 2014), based on analysis of the capsular polysaccharide gene island.

337 Phylogenetically, these isolates clustered together in comparison to other previously reported

338 international ST258 K. pneumoniae isolates (Supplementary Figures S2 \& S3). Analysis of the

339 local ST258 isolates revealed three distinct phylogenetic clades involving patients in 20 different

340 healthcare locations, supported by pairwise SNP distributions and Bayesian Analysis of the

341 Population Structure (Figure 3 \& Supplementary Figure S4). These results formed the basis for

342 ongoing prospective genomic analyses. 


\section{Epidemiological analysis}

345 A total of 57 patients were found to harbour isolates belonging to CC258 from 2012-2015. Most

346 patients presented to the Emergency Department $(n=7)$ or were admitted to hospital $(n=44)$ at the

347 time of initial specimen collection. Thirty-three $(58 \%)$ were male, and the median age of the

348 affected patients was 74 (range 20 - 94; IQR 62-83) (Supplementary Table S5). Clinical infection

349 was suspected in $61 \%$ of the cases, with the most common presentation being urinary tract

350 infection $(n=24 ; 42 \%)$. Sepsis was reported in $63 \%$ of those with infection due to KPC-producing

351 K. pneumoniae. KPC-producing isolates were obtained from patients with clinical infection more

352 commonly during 2012-2014 than 2015, where $>50 \%$ of the KPC-producing isolates identified

353 were colonising or screening isolates (Supplementary Figure S5).

355 Of patients from whom a complete travel history was able to be obtained ( $n=46), 22(48 \%)$ reported overseas travel since 1996, and only eight (14\%) reported travel in the 12 months prior to 357 hospitalisation, strongly reinforcing the suspicion of local transmission of KPC. All 57 patients were found to have been hospitalised in Australian healthcare facilities in the 12 months prior to initial positive specimen collection, with a median length of stay of 61 inpatient days (range 0-181 days; IQR 34-101). Four patients had fewer than ten inpatient days in an Australian healthcare facility during this period, three of whom had recent overseas hospitalisation, for which complete hospitalisation data could not be collected. Additional epidemiological data is detailed in the Supplementary Appendix.

A detailed analysis of patient admission data was undertaken in attempt to identify location or source of acquisition. Forty-one patients ( $72 \%)$ had previously attended one facility (Facility F) in 
367 the 12 months prior to initial identification of a KPC-producing isolate. However, the remaining

36816 patients reported no hospitalisation in this facility, and 18 additional facilities were identified

369 in which two or more patients had been admitted. Three putative transmission networks were

370 identified based on overlapping admissions to the same hospital ward at the same time among

371 patients detected with KPC. It became apparent that due to the sheer number of hospitalisations

372 and the complexity of patient movements, drawing firm conclusions on who transmitted to whom

373 and where transmission had occurred solely from epidemiological data would be difficult (Figure

3744 \& Supplementary Figure S6).

375

376 Combined analysis of CC258 KPC-2-producing K. pneumoniae isolates

377 Despite identification of three distinct phylogenetic clades in the initial genomic analysis (Figure

378 3), we were unable to exactly define where transmission was occurring as the samples were 379 distributed across multiple locations. Of the three clades (designated as genomic cluster A, B and 380 C), cluster $\mathrm{C}$ had the best correlation with an epidemiologically-defined transmission network, 381 comprising isolates from patients in the same healthcare facility at the same time (Figure 4). 382 However, other correlations were not immediately apparent. Cluster A included isolates from three 383 patients in three different facilities that were within the same healthcare network and/or were 384 geographically located in the same region within a $20 \mathrm{~km}$ radius, though were $30 \mathrm{~km}$ away from 385 the fourth patient in a different healthcare network in the cluster. Cluster B included isolates from 386 patients in 12 different locations across the state of Victoria, separated by up to $500 \mathrm{~km}$. Seven 387 isolates were obtained from patients attending local general practice (primary care) clinics. 
389 Using the genomic data to enhance and allow flexibility in the epidemiological data (e.g. including

390 admissions to adjacent wards or admissions separated by a few days as "overlapping"), it became

391 apparent that each of the genomic clusters corresponded to a separate transmission network (Figure

392 5). The largest of these (cluster B) was located at a single institution (Facility F), and as further

393 isolates were identified, two subclusters (B1, B2) were defined. Cluster B2 included isolates

394 arising from suspected transmission in mid-2014 in an inpatient aged care ward, while cluster

395 included isolates from a number of patients who were admitted to Facility $F$ in 2012, though to

396 several different wards. A second transmission network in cluster B1 was identified in another

397 aged care ward in 2015. The predicted transmission network corresponding with cluster C was

398 also confirmed, with the isolate genomes differing by $<5$ SNPs, and the corresponding

399 epidemiological data for the respective patients showing overlapping hospital admissions to a 400 single hospital ward in late 2013 (Figure 6).

401

402 Combining epidemiological and genomic data also revealed secondary transmission events, where 403 patients with undetected colonisation at one hospital were transferred to a second healthcare 404 service, with subsequent diagnosis and onwards transmission. Patients whose isolates were 405 phylogenetically clustered with other isolates from a known transmission network, but who had 406 not previously been admitted to the hospital where transmission was occurring, were flagged as 407 potentially having acquired KPC through a secondary transmission event. Through this, two 408 putative secondary transmission events were later identified from these main transmission 409 networks (see example case study in Figure 6). 


\section{Bayesian evolutionary and transmission analysis}

412 Although from the combined genomic and epidemiological analyses, it appeared that local

413 transmission within separate hospitals was driving the outbreak, local isolates from each cluster

414 were closer to other local isolates (median pairwise SNP distance $=16$; IQR 9-38) than to

415 international ST258 isolates from GenBank (median pairwise SNP distance = 243; IQR 80-258).

416 To investigate the possibility of initial inter-hospital transmission leading to subsequent

417 transmission networks within each hospital, we explored whether Bayesian evolutionary and

418 transmission modelling could be used to provide additional insight.

419

420 From these analyses, genomic clusters $\mathrm{A}$ and $\mathrm{C}$ each appeared to be derived from separate overseas

421 importations of KPC-producing $K$. pneumoniae with subsequent spread within a single hospital or

422 healthcare network rather than inter-hospital transmission, with a most recent common ancestor

423 (MRCA) predicted to have occurred prior to 2010 (median 2007.78; 95\% HPD 2003.30-2011.09)

424 when the first KPC-producing organism was reported in Australia (Coatsworth et al. 2012), and

425 2012, when the first KPC-producing organism was reported in Victoria (Chua et al. 2014) (Figure

426 7). Similarly, the unclustered isolates represented separate individual importations in these

427 analyses, supporting the known history of recent overseas travel for these patients (Supplementary

428 Table S5).

429

430 Cluster B likely arose from another overseas importation (MRCA cluster A and B: median

$4312002.52 ; 95 \%$ HPD 1991.72-2008.79), though the timed phylogeny (Figure 7) suggested strains

432 of KPC-producing K. pneumoniae from cluster B may have been circulating in Victoria since 2011

433 (MRCA cluster B1 and B2: median 2010.59; 95\% HPD 2008.67-2011.86). A number of internal 
434 nodes within cluster B1 were dated to 2012 (Supplementary Figure S8), corresponding with 435 overlapping patient admissions at Facility $\mathrm{F}$ at that time, which affirmed the putative transmission 436 network in Facility F in 2012. In both clusters B1 and B2, many isolates were linked by 437 transmission events that were predicted to have occurred during 2013, despite a decline in new 438 cases detected during this period. Notably, many of the isolates derived from these transmission 439 events were not detected until later, in 2014 or 2015. This supported the epidemiological 440 hypothesis that detection of previously unrecognised colonisation was driving a large proportion 441 of the new cases in 2014 and 2015, rather than new transmission events (Figure 8). For each of the

442 suspected transmission networks identified in the combined outbreak investigation, posterior 443 probability distributions for the MRCA were generally consistent with the epidemiological data 444 (Supplementary Figure S8).

445

446 From the TransPhylo analysis, the mean reproductive number $\left(\mathrm{R}_{0}\right)$ across the outbreak was 447 calculated to be $1.63(\mathrm{SD}=0.13)$, accounting for an average estimated sampling proportion of 0.27 $448 \quad(\mathrm{SD}=0.05)$.

\section{Plasmid analysis}

451 In late 2015, other Enterobacteriaceae carrying bla KPC-2 $_{2}$ were detected, including Citrobacter 452 farmeri, Citrobacter freundii, and Klebsiella oxytoca (Figure 2), which raised concern about KPC453 plasmid spread. Analysis of the de novo assembled draft genomes indicated the bla $_{\mathrm{KPC}-2}$ carrying 454 plasmids in these organisms matched a bla $a_{\mathrm{KPC}-2}$ carrying plasmid found in the locally circulating 455 K. pneumoniae isolates, suggesting inter-species plasmid movement, and subsequent organism 456 transmission. All K. pneumoniae ST258 isolates were found to harbour bla $a_{\mathrm{KPC}-2}$ within the "a" 
457 isoform of the characteristic Tn4401 transposon, and usually on a contig containing sequence

458 encoding the replication regulator RNAI from IncFII(K) plasmids. Analysis of the PacBio

459 reference $K$. pneumoniae genome assembly showed the presence of both IncFIB(pQil) and

460 IncFIB(K) plasmids. The ST258 draft genome assemblies also all contained IncFIB replicons,

461 though the assemblies were inadequate to confirm the plasmid types carrying Tn4401.

462 Chromosomal integration of the Tn4401 within the putative diguanylate cyclase gene, $y c d T$, was

463 found in two epidemiologically linked ST258 K. pneumoniae isolates. One ST258 K. pneumoniae

464 isolate lacked $b l a_{\mathrm{KPC}-2}$, and subsequent repeat susceptibility testing confirmed the isolate to be

465 susceptible to carbapenems. Re-testing of 10 colonies subcultured from the stored glycerol stock

466 for that patient sample found only $7 / 10$ displayed carbapenemase hydrolytic acitivity with the

467 CarbaNP test, indicating in vivo or in vitro loss of the bla $a_{\mathrm{KPC}}$ gene.

468

469 Despite presence of the Tn4401 and IncFII(K) RNAI in the C. farmeri draft assemblies, they 470 lacked the IncFIB replicons detected in the $K$. pneumoniae genomes. To determine if interspecies

471 plasmid transmission had occurred, one C. farmeri isolate underwent PacBio sequencing.

472 Sequence comparisons between the KPC-plasmid genome from this isolate and the KPC-plasmid

473 genome from the K. pneumoniae PacBio reference genome indicated almost identical plasmids.

474 The $C$. farmeri plasmid carried an IncR-type replicon and lacked a Type I restriction modification

475 system, but otherwise comprised an identical sequence to $85 \%$ of the $K$. pneumoniae KPC plasmid

476 following a recombination and inversion event (Figure 9). The C. freundii isolate was also found

477 to have an IncR-type plasmid, but lacked other gene content found in the C. farmeri IncR plasmids,

478 suggesting mobilisation of the Tn4401 transposon. The exact location of $b l a_{\mathrm{KPC}-2}$ and plasmid type

479 of the $C$. freundii and $K$. oxytoca isolates were unable to be determined due to the limitations of 
480 the assembled short-read sequencing data. Phylogenetic analysis of the $C$. farmeri isolates inferred

481 from core genome (chromosomal) SNPs confirmed the isolates were closely related

482 (Supplementary Figure S9).

483

484 Within-host diversity

485 To assess the within-host genomic diversity of KPC-producing Enterobacteriaceae, the isolate

486

487

488

489

490

491

492

493

494

495

496

497

498

499

500

501 genomes from 17 patients who had multiple isolates obtained and sequenced were compared. Of these, one patient had 32 isolates collected from seven samples over an 8-month period from February 2016 to October 2016, including multiple colony sampling from two faecal samples. The other 16 patients had multiple (median = 2; range 2-4) isolates referred to MDU PHL, obtained through recurrent presentations at different healthcare facilities.

Genomic comparisons indicated isolates from the same patient differed by up to 21 SNPs in the core genome. This included the 32 isolates obtained from patient 70 , with several internal lineages emerging from the same common ancestor within that individual (Figure 10). Pangenome analyses of these isolates also demonstrated changes in the accessory genome correlating with the emerging internal lineages in the core genome (chromosomal) phylogeny, and lineage-specific plasmid carriage (Supplementary Figure S10). These changes included subtle differences in plasmid types, acquisition/loss/switching of transposons, and loss/gain of heavy metal operons. There was a poor molecular clock signal for these isolates (root-to-tip divergence $R^{2}=0.026$ ), and no further temporal analyses were undertaken. 
502 When compared to other outbreak isolates, multiple isolates from the same host clustered together 503 within a single subclade in the phylogenetic tree. This supported the concept of a "transmission 504 bottleneck", where despite an assumed diverse within-host population of KPC-producing 505 Enterobacteriaceae, transmission only involved a single isolate, with subsequent within-host 506 evolution following the initial transmission event. This also suggested multiple acquisitions of 507 different strains of KPC-producing K. pneumoniae by the same patient were uncommon among 508 the patient cohort. Of patients with multiple isolates spanning a six-month period, only patients 18 509 and 70 appeared to have isolates from differing subclades, though bootstrap branch support for 510 these subclades was marginal $(0.70-0.75)$.

511

\section{Environmental diversity}

513 A limited number of KPC-producing environmental $K$. pneumoniae isolates obtained at a single 514 time-point from frequently touched surfaces in the room of patient 75 underwent WGS and were 515 compared to the other patient isolates (Figure 10). As expected, the environmental isolates 516 clustered with the clinical isolate from patient 75 , as well as those from patient 72 , who had 517 previously shared a (different) room with patient 75 . Notably, the genomic diversity between these 518 clinical and environmental isolates was less than the within-host diversity seen within patient 70 , 519 supporting the hypotheses that the direction of transmission was from patient 75 to the 520 environment, rather than environment to patient, and that transmission had occurred between 521 patients 72 and 75 when they previously shared a room. 


\section{Infection control investigation}

524 Qualitative interviews were conducted with infection control practitioners at three facilities 525 regarding five transmission networks (four primary clusters of KPC-producing ST258 K.

526 pneumoniae including two secondary transmission events at different locations, in addition to one

527 cluster of KPC-producing C. farmeri) of suspected local transmission involving 17 patients. The

528 majority of transmission events were thought to have occurred due to unrecognised colonisation,

529 and prior to the implementation of contact precautions - single room isolation with en-suite

530 bathroom, with requirements for use of personal protective equipment (gloves and gown) by staff

531 and visitors entering the room. However, two instances of apparent transmission to and from

532 patients under contact precautions were reported. In each of these situations, after being admitted

533 directly into contact precautions due to known colonisation with other multidrug-resistant 534 organisms, a patient acquired a KPC-producing K. pneumoniae isolate that was highly related to 535 other isolates in a genomic cluster ( $<5$ SNPs in the core genome).

536

537 Due to the lack of local guidelines at the time of the outbreak, the management of wards where transmission was found to have occurred was at the discretion of the individual healthcare facilities involved. A retrospective review of the methods used to interrupt transmission identified a number

540 of bundled approaches, with the common measures being contact screening, enhanced cleaning

541 (in contrast to standard cleaning), and isolation of identified cases under contact precautions 542 (Supplementary Table S6).

543

544 Impact of whole-genome sequencing on the outbreak investigation and development of local 545 containment guidelines 
546 In response to the genomic and epidemiological evidence of an evolving outbreak with local

547 transmission, a state-wide management guideline was developed for the surveillance and

548 containment of carbapenemase-producing Enterobacteriaceae incorporating the routine use of

549 WGS to determine relatedness between isolates (Department of Health \& Human Services 2015).

550

551 Several insights were gleaned through the use of genomics that were fundamental to the outbreak 552 investigation and informed development of the guidelines and their focus on hospitals. For 553 example, through the epidemiological investigation, the identification of KPC in previously 554 hospitalised patients suggested nosocomial rather than community transmission, though which

555 hospital transmission was occurring within was not immediately clear, given the number of 556 hospitals and prior overlapping hospitalisations involved. The resolution of the genomic data 557 refined epidemiological hypotheses to pinpoint transmission areas in individual hospitals to focus 558 infection control efforts, with Bayesian analyses of the genomic data also supporting the 559 epidemiological findings. By determining when and where transmission was occurring, it was 560 evident that many new cases were readmissions of patients with previously unrecognised 561 colonisation, rather than new transmission events, making it difficult to "control" the outbreak. 562 This highlighted the need to intensively screen at-risk patient contacts, including flagging those 563 that had been discharged from an affected ward prior to recognition of transmission for screening 564 and pre-emptive isolation upon subsequent hospital presentations. This real-time change-in565 practice was reflected by the increasing proportion of cases identified as "colonisations" rather 566 than "infections" due to intensified screening practices over time (Supplementary Figure S5). 


\section{DISCUSSION}

568 The emergence of carbapenemase producing Enterobacteriaceae (CPE) is a major threat to human

569 health (Australian Commission on Safety and Quality in Health Care 2013; Centers for Disease

570 Control and Prevention 2013; World Health Organization 2014), with significant interventions

571 required at state and national levels to contain the spread once established (Schwaber \& Carmeli

572 2014). Here, we describe the largest outbreak of CPE reported in Australia, and demonstrate how

573 a combined genomics and epidemiological investigation delineated the outbreak into five separate

574 nosocomial transmission networks (four clusters of $K$. pneumoniae and one cluster of C. farmeri)

575 across the state over four years, resulting in targeted interventions for each transmission area. The

576 use of WGS for outbreak analysis has been well established, including for transmission of

577 multidrug resistant hospital pathogens (Palmore \& Henderson 2013; Snitkin et al. 2012), but these

578 studies have been predominantly small scale, single institution studies, and often retrospective.

579 Genomics has rarely been used prospectively and in real-time during a complex multi-institutional

580 outbreak requiring a coordinated state-wide public health response. Due to prolonged colonisation,

581 many CPE cases linked to hospitals with suspected transmission were identified through other

582 healthcare facilities, and some through general practice. With the number of potential transmission

583 opportunities in retrospective hospitalisation data from all facilities, the epidemiological

584 investigation would have been difficult to interpret and translate into focussed interventions

585 without the resolution offered by genomics. Similarly, accurate interpretation of the genomic data

586 would have been difficult without the supporting epidemiologic data. As others have pointed out,

587 the two must go hand-in-hand (Tong 2013). By integrating our detailed epidemiological

588 investigation with genomic analyses, we were able to refine our hypotheses, and coordinate an 
589 effective public health response to target areas with ongoing transmission, emphasising the ability 590 of WGS to enhance surveillance systems and outbreak investigations.

591

592 Despite integrating genomic data with detailed epidemiological information, we still encountered 593 several challenges. Accurate inference of plasmid transmission can be challenging from short-read Illumina sequencing data, with repetitive elements such as insertion sequences that accompany 595 mobile resistance elements frequently confounding short-read assemblers and read-aligners (Conlan et al. 2014). As we demonstrated here and as reported by others (Conlan et al. 2014), longread sequencing technology such as Pacific Biosciences single-molecule sequencing can be highly useful in resolving and tracking the diversity of plasmids carrying carbapenemases and other resistance genes, although we could only sequence a limited representation of the outbreak isolates due to cost limitations. The data gained from these completed genomes were invaluable. The use of a local internal reference provided additional confidence in SNP calling among highly clonal 602 isolates. We were able to compare completely assembled plasmids between different species, which otherwise would not have been possible. Many isolates in the outbreak were hypothesised to carry bla $_{\mathrm{KPC}-2}$ on either an $\operatorname{IncFIB}(\mathrm{K})$ or $\operatorname{IncFIB(pQil)~plasmid,~with~long-read~sequencing~}$ showing it was possible for isolates to harbour multiple plasmids of the same incompatibility 606 group. As we found in two isolates and as others have recently reported (Mathers et al. 2017), 607 chromosomal integration of Tn4401 also occasionally occurs. The movement of these resistance 608 elements adds complexity to understanding transmission dynamics using genomics, and highlights 609 the limitations of short-read sequencing data to fully characterise these elements. 
611 It is increasingly evident that the subtly diverse populations of a single clonal type due to within-

612 host evolution (Golubchik et al. 2013), and more diverse populations from transmission of mixed

613 infections (Eyre et al. 2013; Hatherell et al. 2016), can impair accurate reconstruction of

614 transmission pathways from genomic data (Worby et al. 2014). In our study, we found some

615 individuals not only had different plasmid variants, but also had distinct within-host evolutionary

616 lineages, indicating a complex evolutionary history of transmission, within-host evolution, and

617 plasmid movement, mirroring recent reports in other patients with prolonged KPC colonisation

618 (Conlan et al. 2016). However, measuring this diversity is difficult in outbreak investigations.

619 Although single-colony sampling and sequencing arguably provides the most informative data, it

620 may be subject to colony selection bias, so some uncertainty remains. This can be offset by

621 sampling more colonies, but the time and cost of sequencing each colony can quickly become

622 prohibitive in an outbreak investigation and real-world public health environment. Although some

623 have attempted to detect the presence of mixed infections in short-read datasets through analysis

624 of short-read mapping from deep sequencing (Eyre et al. 2013), this relies on WGS performed on

625 a sweep of a primary culture plate, introducing the potential for exogenous DNA in the dataset,

626 and becomes considerably more complicated with fluxes in accessory genome content. As we

627 found, bla $a_{\mathrm{KPC}}-$ plasmids can be lost even through laboratory passage of stored isolates. Even if the

628 within-host genomic diversity can be captured, incorporating these data into models can be

629 difficult. Recent attempts to account for elements such as within-host diversity and unsampled data

630 in reconstructing transmission trees have proved successful in some situations (Didelot et al. 2017;

631 Jombart et al. 2014; Klinkenberg et al. 2017; Ypma et al. 2013). However, each of these make

632 several assumptions, such as the presence of a complete transmission bottleneck that does not

633 allow for repeated acquisition, a constant reproduction number across the outbreak, few or no 
634 unsampled cases, and that genetic variation is accumulated in pathogens in a clock-like fashion.

635 The within-host diversity and horizontal transmission of mobile genetic elements in outbreak

636 investigations complicates analyses further, and consequently, accurate reconstruction of exact

637 transmission routes remains difficult for large and moderate-sized CPE outbreaks.

638

639 Our study has some limitations and points of note. Firstly, although most patient isolates had a 640 clear epidemiological link to other isolates within the defined genomic clusters, not every isolate 641 was able to be linked to another patient with KPC or a history of recent overseas travel, indicating 642 a larger pool of unrecognised colonisation, though influenced in part by differing stringency in 643 contact screening and data collection. For example, no patient in cluster A reported overseas travel 644 since 1996, when KPC was first detected. Furthermore, although these patients were located at 645 healthcare facilities in the same geographical region of Victoria and part of the same healthcare 646 service, they did not have any overlapping inpatient admissions. Thus, it is likely that there are 647 other individuals or environmental sources that serve as intermediary reservoirs of isolates not 648 captured in our sampling, that facilitate ongoing transmission. Although environmental and contact 649 screening was initially performed using methods published by others (Centers for Disease Control 650 and Prevention 2015), based on our data, the optimal approaches are still not well understood. 651 Secondly, although the outbreak has slowed, cases are still emerging despite the extensive 652 investigation and interventions implemented by the individual healthcare services. However, as 653 shown in our analyses, many of these may be unrecognised colonisation following previous 654 exposure rather than new transmission events. As a result of this knowledge, through the 655 development of our guidelines, we have redirected infection control resources into intensive 656 screening to identify individuals with asymptomatic colonisation, focussing efforts on healthcare 
657 facilities with ongoing transmission. Thirdly, as discussed above, capturing within-host population

658 genomic diversity and reconstructing entire plasmid genomes from each clinical sample through

659 sequencing would further enhance future outbreak investigations, but are currently limited by the

660 cost of performing such analyses routinely. Sequencing cost and accuracy limitations also prohibit

661 the routine use of more rapid sequencing technologies, such as PacBio SMRT and Oxford

662 Nanopore sequencing, which could potentially provide more rapid results than Illumina

663 sequencing to inform infection control decisions for prospectively sequenced isolates, though in

664 the context of an outbreak spanning several years, turn-around times for Illumina sequencing can

665 still be considered "real-time". Finally, although we conducted transmission inference analyses,

666 these methods require further validation for routine implementation in outbreak investigations of

667 multidrug-resistant pathogens. For example, we used TransPhylo in preference to other

668 transmission modelling software as it did not require a high sampling proportion, and it provided

669 the ability to account for and estimate the proportion of missing cases. However, it is difficult to

670 know the true accuracy of these estimates, as even transmission chains arising from densely

671 sampled wards were predicted to have unsampled cases by TransPhylo. Several possibilities to

672 explain these unsampled cases include the limited sensitivity of the screening methods used, and

673 the role of staff, visitors and the hospital environment in reconstructing transmission networks.

674 Additionally, although transmission chains were predicted by TransPhylo, the resolution of the

675 epidemiological data was insufficient to confirm the precise sequence of transmission events in a

676 complex outbreak, with multiple potential pathways. Robust validation of these transmission

677 models is required before being applied in outbreak investigations to determine who infected

678 whom - results that may subsequently have considerable ethical or legal implications.

679 


\section{CONCLUSIONS}

681 Our study reports a real-world prospective utilisation of WGS, including the difficulties 682 encountered, to enhance a complex epidemiological investigation in real-time for an important 683 pathogen. In contrast to most reported outbreaks of CPE within a single institution, our outbreak 684 demanded a coordinated public health response. Previously the domain and responsibility of 685 individual hospitals and healthcare facilities, the spread of CPE has become a public health issue. 686 Through our experience, WGS has since been incorporated into our state guidelines for the 687 management of CPE (Department of Health \& Human Services 2015). Developed during the 688 outbreak investigation, this system entails routine WGS of all suspected CPE isolates with concurrent epidemiological investigation to allow prospective, centralised comparison of isolates

690 and epidemiological data from multiple health services. In turn, this enables identification of 691 potential transmission events between patients geographically and temporally dispersed at 692 identification, translating into focussed interventions at the designated transmission locations. For 693 such extensively drug-resistant organisms with limited treatment options, all feasible interventions 694 towards reducing the early burden of disease are warranted. 


\section{ACKNOWLEDGEMENTS}

696 We wish to acknowledge and thank the referring clinical microbiology laboratories for facilitating 697 transfer of isolates to MDU PHL, and the hospital infection control and infectious diseases staff 698 who assisted and facilitated epidemiological data collection from the corresponding patients. We 699 also thank Dr Xavier Didelot, Imperial College London for his advice and assistance with 700 TransPhylo, and Dr Sally Partridge, University of Sydney for her expertise in Gram-negative 701 plasmids.

702

703 


\section{REFERENCES}

705

706

707

708

709

710

711

712

713

714

715

716

717

718

719

720

721

722

723

724

725

726

727

Australian Commission on Safety and Quality in Health Care. 2013. Recommendations for the control of multi-drug resistant Gram-negatives: carbapenem resistant Enterobacteriaceae. Sydney: Australian Commission on Safety and Quality in Health Care. Available from: https://www.safetyandquality.gov.au/publications/mrgn-guidelines-enterobacteriacea/.

Australian Group on Antimicrobial Resistance. 2014. The evolution of carbapenemases in Enterobacteriaceae in Australia. Available from: http://www.agargroup.org/surveys.

Bankevich A, Nurk S, Antipov D, Gurevich AA, Dvorkin M, Kulikov AS, Lesin VM, Nikolenko SI, Pham S, Prjibelski AD, Pyshkin AV, Sirotkin AV, Vyahhi N, Tesler G, Alekseyev MA, and Pevzner PA. 2012. SPAdes: a new genome assembly algorithm and its applications to single-cell sequencing. J Comput Biol 19:455-477.

Blyth CC, Pereira L, and Goire N. 2014. New Delhi metallo-beta-lactamase-producing enterobacteriaceae in an Australian child who had not travelled overseas. Med J Aust 200:386.

Bolger AM, Lohse M, and Usadel B. 2014. Trimmomatic: a flexible trimmer for Illumina sequence data. Bioinformatics 30:2114-2120.

Bouckaert R, Heled J, Kuhnert D, Vaughan T, Wu CH, Xie D, Suchard MA, Rambaut A, and Drummond AJ. 2014. BEAST 2: a software platform for Bayesian evolutionary analysis. PLoS Comput Biol 10:e1003537.

Camacho C, Coulouris G, Avagyan V, Ma N, Papadopoulos J, Bealer K, and Madden TL. 2009. BLAST+: architecture and applications. BMC Bioinformatics 10:421.

Carattoli A, Zankari E, Garcia-Fernandez A, Voldby Larsen M, Lund O, Villa L, Moller Aarestrup F, and Hasman H. 2014. In silico detection and typing of plasmids using PlasmidFinder and plasmid multilocus sequence typing. Antimicrob Agents Chemother 58:3895-3903. 
728 Carter GP, Buultjens AH, Ballard SA, Baines SL, Tomita T, Strachan J, Johnson PD, Ferguson JK,

729

730

731

732

733

734

735

736

737

738

739

740

741

742

743

744

745

746

747

748

749

750

751

752 753

Seemann T, Stinear TP, and Howden BP. 2016. Emergence of endemic MLST non-typeable vancomycin-resistant Enterococcus faecium. $J$ Antimicrob Chemother 71:3367-3371.

Centers for Disease Control and Prevention, US Department of Health and Human Services. 2013. Antibiotic resistance threats in the United States, 2013. Available from: https://www.cdc.gov/drugresistance/threat-report-2013/index.html.

Centers for Disease Control and Prevention, US Department of Health and Human Services. 2015. Facility Guidance for Control of Carbapenem-resistant Enterobacteriaceae (CRE). Available from: https://www.cdc.gov/hai/organisms/cre/cre-toolkit/index.html.

Chang LW, Buising KL, Jeremiah CJ, Cronin K, Poy Lorenzo YS, Howden BP, Kwong J, Cocks J, Blood A, Greenough J, and Waters MJ. 2015. Managing a nosocomial outbreak of carbapenem-resistant Klebsiella pneumoniae: an early Australian hospital experience. Intern Med J 45:1037-1043.

Chen L, Chavda KD, Mediavilla JR, Jacobs MR, Levi MH, Bonomo RA, and Kreiswirth BN. 2012. Partial excision of blaKPC from Tn4401 in carbapenem-resistant Klebsiella pneumoniae. Antimicrob Agents Chemother 56:1635-1638.

Cheng L, Connor TR, Siren J, Aanensen DM, and Corander J. 2013. Hierarchical and spatially explicit clustering of DNA sequences with BAPS software. Mol Biol Evol 30:1224-1228.

Chua KY, Grayson ML, Burgess AN, Lee JY, and Howden BP. 2014. The growing burden of multidrugresistant infections among returned Australian travellers. Med J Aust 200:116-118.

Coatsworth NR, Huntington PG, Hardiman RP, Hudson BJ, and Fernandes CJ. 2012. A case of carbapenemase-producing Klebsiella pneumoniae in Australia. Pathology 44:42-44.

Conlan S, Park M, Deming C, Thomas PJ, Young AC, Coleman H, Sison C, Program NCS, Weingarten RA, Lau AF, Dekker JP, Palmore TN, Frank KM, and Segre JA. 2016. Plasmid Dynamics in KPC-Positive Klebsiella pneumoniae during Long-Term Patient Colonization. MBio 7.

Conlan S, Thomas PJ, Deming C, Park M, Lau AF, Dekker JP, Snitkin ES, Clark TA, Luong K, Song Y, Tsai YC, Boitano M, Dayal J, Brooks SY, Schmidt B, Young AC, Thomas JW, Bouffard GG, 
754

755

756

757

758

759

760

761

762

763

764

765

766

767

768

769

770

771

772

773

774

775

776

777

Blakesley RW, Program NCS, Mullikin JC, Korlach J, Henderson DK, Frank KM, Palmore TN, and Segre JA. 2014. Single-molecule sequencing to track plasmid diversity of hospital-associated carbapenemase-producing Enterobacteriaceae. Sci Transl Med 6:254ra126.

Deleo FR, Chen L, Porcella SF, Martens CA, Kobayashi SD, Porter AR, Chavda KD, Jacobs MR, Mathema B, Olsen RJ, Bonomo RA, Musser JM, and Kreiswirth BN. 2014. Molecular dissection of the evolution of carbapenem-resistant multilocus sequence type 258 Klebsiella pneumoniae. Proc Natl Acad Sci U S A 111:4988-4993.

Department of Health \& Human Services, Victoria State Government. 2015. Victorian guidelines on carbapenemase-producing Enterobacteriaceae. Melbourne: Victoria State Government. Available from: https://www2.health.vic.gov.au/about/publications/policiesandguidelines/carbapenemaseproducing-enterobacteriaceae-guidelines.

Didelot X, Fraser C, Gardy J, and Colijn C. 2017. Genomic Infectious Disease Epidemiology in Partially Sampled and Ongoing Outbreaks. Mol Biol Evol 34:997-1007.

Didelot X, and Wilson DJ. 2015. ClonalFrameML: efficient inference of recombination in whole bacterial genomes. PLoS Comput Biol 11:e1004041.

Drummond AJ, Ho SY, Phillips MJ, and Rambaut A. 2006. Relaxed phylogenetics and dating with confidence. PLoS Biol 4:e88.

Espedido BA, Steen JA, Ziochos H, Grimmond SM, Cooper MA, Gosbell IB, van Hal SJ, and Jensen SO. 2013. Whole genome sequence analysis of the first Australian OXA-48-producing outbreakassociated Klebsiella pneumoniae isolates: the resistome and in vivo evolution. PLoS One 8:e59920.

Eyre DW, Cule ML, Griffiths D, Crook DW, Peto TE, Walker AS, and Wilson DJ. 2013. Detection of mixed infection from bacterial whole genome sequence data allows assessment of its role in Clostridium difficile transmission. PLoS Comput Biol 9:e1003059. 
778 Falagas ME, Lourida P, Poulikakos P, Rafailidis PI, and Tansarli GS. 2014. Antibiotic treatment of

779

780

781

782

783

784

785

786

787

788

789

790

791

792

793

794

795

796

797

798

799

800

801

802

803 infections due to carbapenem-resistant Enterobacteriaceae: systematic evaluation of the available evidence. Antimicrob Agents Chemother 58:654-663.

Fernando GA, Collignon PJ, and Bell JM. 2010. A risk for returned travellers: the "post-antibiotic era". Med J Aust 193:59.

Gilchrist CA, Turner SD, Riley MF, Petri WA, Jr., and Hewlett EL. 2015. Whole-genome sequencing in outbreak analysis. Clin Microbiol Rev 28:541-563.

Golubchik T, Batty EM, Miller RR, Farr H, Young BC, Larner-Svensson H, Fung R, Godwin H, Knox K, Votintseva A, Everitt RG, Street T, Cule M, Ip CL, Didelot X, Peto TE, Harding RM, Wilson DJ, Crook DW, and Bowden R. 2013. Within-host evolution of Staphylococcus aureus during asymptomatic carriage. PLoS One 8:e61319.

Hasegawa M, Kishino H, and Yano T. 1985. Dating of the human-ape splitting by a molecular clock of mitochondrial DNA. J Mol Evol 22:160-174.

Hatherell HA, Colijn C, Stagg HR, Jackson C, Winter JR, and Abubakar I. 2016. Interpreting whole genome sequencing for investigating tuberculosis transmission: a systematic review. BMC Med $14: 21$.

Jiang Y, Wei Z, Wang Y, Hua X, Feng Y, and Yu Y. 2015. Tracking a hospital outbreak of KPCproducing ST11 Klebsiella pneumoniae with whole genome sequencing. Clin Microbiol Infect 21:1001-1007.

Jombart T, Cori A, Didelot X, Cauchemez S, Fraser C, and Ferguson N. 2014. Bayesian reconstruction of disease outbreaks by combining epidemiologic and genomic data. PLoS Comput Biol 10:e1003457.

Kanamori H, Parobek CM, Juliano JJ, van Duin D, Cairns BA, Weber DJ, and Rutala WA. 2017. A Prolonged Outbreak of KPC-3-Producing Enterobacter cloacae and Klebsiella pneumoniae Driven by Multiple Mechanisms of Resistance Transmission at a Large Academic Burn Center. Antimicrob Agents Chemother 61. 
804 Kitchel B, Rasheed JK, Patel JB, Srinivasan A, Navon-Venezia S, Carmeli Y, Brolund A, and Giske CG.

805

806

807

808

809

810

811

812

813

814

815

816

817

818

819

820

821

822

823

824

825

826

827

828 2009. Molecular epidemiology of KPC-producing Klebsiella pneumoniae isolates in the United States: clonal expansion of multilocus sequence type 258. Antimicrob Agents Chemother 53:3365-3370.

Klinkenberg D, Backer JA, Didelot X, Colijn C, and Wallinga J. 2017. Simultaneous inference of phylogenetic and transmission trees in infectious disease outbreaks. PLoS Comput Biol 13:e1005495.

Kotsanas D, Wijesooriya WR, Korman TM, Gillespie EE, Wright L, Snook K, Williams N, Bell JM, Li HY, and Stuart RL. 2013. "Down the drain": carbapenem-resistant bacteria in intensive care unit patients and handwashing sinks. Med J Aust 198:267-269.

Kwong JC, McCallum N, Sintchenko V, and Howden BP. 2015. Whole genome sequencing in clinical and public health microbiology. Pathology 47:199-210.

Kwong JC, Mercoulia K, Tomita T, Easton M, Li HY, Bulach DM, Stinear TP, Seemann T, and Howden BP. 2016. Prospective Whole-Genome Sequencing Enhances National Surveillance of Listeria monocytogenes. J Clin Microbiol 54:333-342.

Marsh JW, Krauland MG, Nelson JS, Schlackman JL, Brooks AM, Pasculle AW, Shutt KA, Doi Y, Querry AM, Muto CA, and Harrison LH. 2015. Genomic Epidemiology of an EndoscopeAssociated Outbreak of Klebsiella pneumoniae Carbapenemase (KPC)-Producing K. pneumoniae. PLoS One 10:e144310.

Mathers AJ, Stoesser N, Chai W, Carroll J, Barry K, Cherunvanky A, Sebra R, Kasarskis A, Peto TE, Walker AS, Sifri CD, Crook DW, and Sheppard AE. 2017. Chromosomal Integration of the Klebsiella pneumoniae Carbapenemase Gene, blaKPC, in Klebsiella Species Is Elusive but Not Rare. Antimicrob Agents Chemother 61.

Mathers AJ, Stoesser N, Sheppard AE, Pankhurst L, Giess A, Yeh AJ, Didelot X, Turner SD, Sebra R, Kasarskis A, Peto T, Crook D, and Sifri CD. 2015. Klebsiella pneumoniae carbapenemase 
(KPC)-producing K. pneumoniae at a single institution: insights into endemicity from wholegenome sequencing. Antimicrob Agents Chemother 59:1656-1663.

831

Munoz-Price LS, Poirel L, Bonomo RA, Schwaber MJ, Daikos GL, Cormican M, Cornaglia G, Garau J, Gniadkowski M, Hayden MK, Kumarasamy K, Livermore DM, Maya JJ, Nordmann P, Patel JB, Paterson DL, Pitout J, Villegas MV, Wang H, Woodford N, and Quinn JP. 2013. Clinical epidemiology of the global expansion of Klebsiella pneumoniae carbapenemases. Lancet Infect Dis 13:785-796.

Naas T, Cuzon G, Truong HV, and Nordmann P. 2012. Role of ISKpn7 and deletions in blaKPC gene expression. Antimicrob Agents Chemother 56:4753-4759.

Naas T, Cuzon G, Villegas MV, Lartigue MF, Quinn JP, and Nordmann P. 2008. Genetic structures at the origin of acquisition of the beta-lactamase bla KPC gene. Antimicrob Agents Chemother 52:12571263.

Nordmann P, Poirel L, and Dortet L. 2012. Rapid detection of carbapenemase-producing Enterobacteriaceae. Emerg Infect Dis 18:1503-1507.

Page AJ, Cummins CA, Hunt M, Wong VK, Reuter S, Holden MT, Fookes M, Falush D, Keane JA, and Parkhill J. 2015. Roary: rapid large-scale prokaryote pan genome analysis. Bioinformatics 31:3691-3693.

Page AJ, Taylor B, Delaney AJ, Soares J, Seemann T, Keane JA, and Harris SR. 2016. SNP-sites: rapid efficient extraction of SNPs from multi-FASTA alignments. Microb Genom 2:e000056.

Palmore TN, and Henderson DK. 2013. Managing transmission of carbapenem-resistant enterobacteriaceae in healthcare settings: a view from the trenches. Clin Infect Dis 57:1593-1599.

Peleg AY, Franklin C, Bell JM, and Spelman DW. 2005. Dissemination of the metallo-beta-lactamase gene blaIMP-4 among gram-negative pathogens in a clinical setting in Australia. Clin Infect Dis 41:1549-1556. 
853 Poirel L, Lagrutta E, Taylor P, Pham J, and Nordmann P. 2010. Emergence of metallo-beta-lactamase

854 NDM-1-producing multidrug-resistant Escherichia coli in Australia. Antimicrob Agents

$855 \quad$ Chemother 54:4914-4916.

856 Price MN, Dehal PS, and Arkin AP. 2010. FastTree 2--approximately maximum-likelihood trees for large $857 \quad$ alignments. PLoS One 5:e9490.

858 Queenan AM, and Bush K. 2007. Carbapenemases: the versatile beta-lactamases. Clin Microbiol Rev $859 \quad 20: 440-458$, table of contents.

860 Rambaut A, Lam TT, Max Carvalho L, and Pybus OG. 2016. Exploring the temporal structure of 861 heterochronous sequences using TempEst (formerly Path-O-Gen). Virus Evol 2:vew007.

862 Ruppe E, Olearo F, Pires D, Baud D, Renzi G, Cherkaoui A, Goldenberger D, Huttner A, Francois P, 863 Harbarth S, and Schrenzel J. 2017. Clonal or not clonal? Investigating hospital outbreaks of KPCproducing Klebsiella pneumoniae with whole-genome sequencing. Clin Microbiol Infect. carbapenem-resistant enterobacteriaceae. Clin Infect Dis 58:697-703.

Seemann T. 2014. Prokka: rapid prokaryotic genome annotation. Bioinformatics 30:2068-2069.

Sidjabat H, Nimmo GR, Walsh TR, Binotto E, Htin A, Hayashi Y, Li J, Nation RL, George N, and

Sidjabat HE, Kennedy K, Silvey A, Collignon P, and Paterson DL. 2013. Emergence of bla(OXA-181)Paterson DL. 2011. Carbapenem resistance in Klebsiella pneumoniae due to the New Delhi carrying ColE plasmid in Klebsiella pneumoniae in Australia. Int J Antimicrob Agents 41:294296.

Snitkin ES, Zelazny AM, Thomas PJ, Stock F, Group NCSP, Henderson DK, Palmore TN, and Segre JA. 2012. Tracking a hospital outbreak of carbapenem-resistant Klebsiella pneumoniae with wholegenome sequencing. Sci Transl Med 4:148ra116.

Stamatakis A. 2014. RAxML version 8: a tool for phylogenetic analysis and post-analysis of large phylogenies. Bioinformatics 30:1312-1313. 
879 Tai AY, Stuart RL, Sidjabat HE, Lemoh CN, Rogers BA, Graham M, Paterson DL, and Korman TM. 880 2015. Local acquisition and nosocomial transmission of Klebsiella pneumoniae harbouring the 881 blaNDM-1 gene in Australia. Med J Aust 202:270-272.

882 Tenover FC, Canton R, Kop J, Chan R, Ryan J, Weir F, Ruiz-Garbajosa P, LaBombardi V, and Persing 883 DH. 2013. Detection of colonization by carbapenemase-producing Gram-negative Bacilli in 884 patients by use of the Xpert MDRO assay. J Clin Microbiol 51:3780-3787.

885 Tong SY. 2013. Genomic polish for shoe-leather epidemiology. Nat Rev Microbiol 11:8.

886 Tzouvelekis LS, Markogiannakis A, Psichogiou M, Tassios PT, and Daikos GL. 2012. Carbapenemases 887 in Klebsiella pneumoniae and other Enterobacteriaceae: an evolving crisis of global dimensions. Clin Microbiol Rev 25:682-707.

889

890

891

892

893

894

895

896

897

898

899

900

901

902

Walker BJ, Abeel T, Shea T, Priest M, Abouelliel A, Sakthikumar S, Cuomo CA, Zeng Q, Wortman J, Young SK, and Earl AM. 2014. Pilon: an integrated tool for comprehensive microbial variant detection and genome assembly improvement. PLoS One 9:e112963.

Weterings V, Zhou K, Rossen JW, van Stenis D, Thewessen E, Kluytmans J, and Veenemans J. 2015. An outbreak of colistin-resistant Klebsiella pneumoniae carbapenemase-producing Klebsiella pneumoniae in the Netherlands (July to December 2013), with inter-institutional spread. Eur J Clin Microbiol Infect Dis 34:1647-1655.

Worby CJ, Lipsitch M, and Hanage WP. 2014. Within-host bacterial diversity hinders accurate reconstruction of transmission networks from genomic distance data. PLoS Comput Biol 10:e1003549.

World Health Organization. 2014. Antimicrobial resistance: global report on surveillance. Available from: http://www.who.int/drugresistance/documents/surveillancereport/en/.

Ypma RJ, van Ballegooijen WM, and Wallinga J. 2013. Relating phylogenetic trees to transmission trees of infectious disease outbreaks. Genetics 195:1055-1062. 
903 Zimmerman FS, Assous MV, Bdolah-Abram T, Lachish T, Yinnon AM, and Wiener-Well Y. 2013.

904 Duration of carriage of carbapenem-resistant Enterobacteriaceae following hospital discharge. $\mathrm{Am}$ $905 \quad$ J Infect Control 41:190-194.

906

907

908 
909

910

911

912

913

914

915

916

917

918

919

920

921

922

923

924

925

926

927

928

929

930

931

\section{FIGURE LEGENDS}

\section{Figure 1: Workflow summary diagram of methods used in this study.}

Microbiology methods (orange), bioinformatic methods (blue), and epidemiological methods (green) used to generate data for analysis and reporting to public health authorities (purple) are shown. Results of combined prospective epidemiological and genomic analyses performed iteratively during the outbreak were reported to the Department of Health and Human Services in real-time. $\mathrm{CPE}=$ carbapenemase-producing Enterobacteriaceae; KPC $=$ Klebsiella pneumoniae carbapenemase; MALDI-TOF = matrix-assisted laser desorption/ionisation time-of-flight; PCR = polymerase chain reaction; WGS $=$ whole-genome sequencing; $\mathrm{SNP}=$ single nucleotide polymorphism; AMR = antimicrobial resistance; MLST $=$ multi-locus sequence typing.

\section{Figure 2: Incidence of new KPC-producing Enterobacteriaceae cases referred to MDU PHL,} 2012-2015.

Blocks are coloured by the species and KPC allele of the referred isolates. Repeated detections of KPC-producing isolates from the same patient have been excluded.

Figure 3: The initial maximum likelihood phylogenetic tree comprised three dominant clades.

The tree includes 29 ST258 K. pneumoniae isolates collected from 29 patients prior to June 2014, with external nodes coloured according to healthcare facility at time of sample collection. Recurrent isolates from each patient have been excluded. Multiple patients in Facility A (purple) and Facility F (orange) were colonised/infected with KPC-producing K. pneumoniae, 
932 corresponding to known previous outbreaks in those hospitals. Support values (\%) from 1000 933 bootstrap replicates are shown for major branches. Major phylogenetic clades have been labelled

934 cluster A (green shading), B (orange shading), and C (purple shading) in the order that the clades

935 emerged, with the larger clade B comprising two subclades, B1 and B2. Corresponding clusters

936 identified through Bayesian Analysis of the Population Structure (BAPS) are also shown. The tree

937 was rooted using an outgroup isolate (K. pneumoniae NJST258_1, GenBank accession:

938 CP006923.1; not shown in the tree) from a different ST258 clade.

Figure 4: Epidemiological links based on overlapping patient admissions were unable to resolve where and when transmission was occurring for most isolates.

942 Links in the above network connect patients based on overlapping patient admissions in the same 943 hospital ward at the same time (minimum of one day overlap). Links that occurred after detection

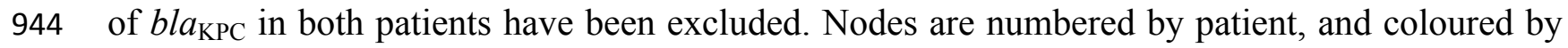
945 healthcare facility (as in Figure 3) at the time of sample collection. The epidemiological network 946 connecting patients $22,23,24$ and 33 correlates with the closely related genomes in Cluster C 947 (Figure 3).

948

949 Figure 5: Combined analysis, with genomic relationships between isolates overlaid upon 950 epidemiological data, delineated multiple transmission networks.

951 A maximum likelihood phylogenetic tree is shown on the left, labelled with major genomic clusters 952 and supporting branch bootstrap values (\%) from 1000 replicates for major branches. Nodes of the 953 tree are coloured by healthcare facility at the time of sample collection as in Figures 3 and 4. 954 Coloured horizontal bars on the right indicate healthcare facility admissions over time (x axis), 
955 with different colours representing different healthcare networks. Black diamonds $(\oplus)$ indicate

956 first detection of KPC for each patient.

957

958 Figure 6: Combined analysis of genomic and epidemiological data of the cluster C network identified secondary transmission.

960 Example case study: Patients 22 and 23 both reported overseas hospitalisation in the 12 months 961 prior to first detection of KPC - patient 22 in Vietnam for spinal surgery following a motor vehicle 962 accident, and patient 23 in Greece for stem cell therapy. Both patients had undergone rectal 963 screening on admission to Ward 1 in Facility A, with patient 22 being placed in intensive contact 964 precautions for the duration of his hospital admissions after isolating another multidrug-resistant 965 organism, though was required to use shared bathroom facilities with patients in the adjacent room. 966 Having required treatment with meropenem for both hospital-acquired pneumonia and a surgical

967 wound infection, patient 22 was later diagnosed with a KPC-producing $K$. pneumoniae indwelling 968 catheter-associated urinary infection in January 2014. Twelve days later, patient 23 was 969 subsequently found to have a polymicrobial sacral wound infection, with cultures including KPC970 producing $K$. pneumoniae from sacral tissue. In response to this, all patients on the ward who had 971 been admitted to the same room and/or shared bathroom facilities with patients 22 and 23 were

972 screened, with the subsequent identification of patient 24. Alerts were placed on the records of 973 patients meeting the criteria who had been previously discharged. Environmental screening of the 974 rooms and bathrooms was conducted, with no KPC-producing organisms identified, and extended 975 bleach cleaning with changes of curtains, chairs and other furnishings was conducted for the entire 976 ward. 
978 Patient 33 was also admitted to Ward 1 in Facility A in February 2014, subsequent to the

979 identification of KPC-2 in patients 22, 23 and 24. This patient was not screened as he had not been

980 admitted to the same room, nor had he shared a bathroom with the identified cases. He also

981 reported no recent history of overseas travel. However, he was identified in July 2014 through

982 routine screening at Facility A following transfer from Ward 3, Facility U, located 25 km away. A

983 KPC-producing isolate from Patient 39 was identified in September 2014, and although the isolate 984 genomically clustered with isolates from Patients 22, 23, 24 and 33 identified at Facility A, she 985 had no previous presentations to that healthcare facility. However, immediately prior to 986 identification of KPC, she had also been in Facility U on Ward 3, though she was admitted there 98713 days after Patient 33's discharge. Given this was the only plausible epidemiological link to the 988 other cluster C patients, secondary transmission was presumed to have occurred in Ward 3, Facility 989 U.

990

991

Figure 7: Bayesian evolutionary analysis indicates each of the phylogenetic clades corresponding to the genomic clusters emerged prior to the detection of KPC in Victoria.

993 A maximum clade credibility timed phylogeny from Bayesian evolutionary analysis of local CC258 K. pneumoniae isolate genomes is shown, with median node heights displayed. The thin red bars indicate 95\% highest posterior density (HPD) intervals for the most recent common ancestor (MRCA) for major clades and defined genomic clusters (indicated on the right). The shaded grey region indicates the recent period when KPC isolates were detected in Victoria.

998

999

Figure 8: An inferred transmission tree shows that undetected colonisation was significant in propagating the outbreak. 
1001 Solid nodes represent the posterior mean time of KPC acquisition by individuals and are coloured

1002 by the corresponding genomic cluster, with empty circles representing inferred unsampled 1003 individuals contributing to the transmission tree. Braches are shaded by number of missing links 1004 in the transmission tree, with lighter branches representing increasing numbers of missing links 1005 implicated.

1006

1007 Figure 9: KPC plasmids from the $C$. farmeri isolates were almost identical to KPC plasmids 1008 from K. pneumoniae outbreak isolates, despite differing replication proteins.

1009 BLAST comparison between an IncFIB (pQIL-like) plasmid genome from a ST258 Klebsiella 1010 pneumoniae isolate, AUSMDU00008079 (above), and an IncR plasmid genome from a 1011 Citrobacter farmeri isolate, AUSMDU00008141 (below), from the outbreak. The grey shading 1012 indicates corresponding DNA regions of high nucleotide identity transcribed in opposing 1013 directions, with the Tn4401 transposon harbouring bla $a_{\mathrm{KPC}-2}$ highlighted in red. The plasmid 1014 genomes have been orientated to their respective replicons and downstream plasmid partitioning 1015 genes, parA and parB.

1016

1017 Figure 10: Genomes of isolates from the same host group together in the phylogeny.

1018 Maximum likelihood tree of the study isolates with additional isolates obtained up to 1 November 10192016 included. Multiple isolates from the same patient have been coloured by patient, with the 1020 accompanying graph indicating the collection dates for the corresponding isolates. Thirty-two 1021 isolates from six clinical samples obtained from patient 70 (blue) over 8 months have been 1022 highlighted to illustrate the within-host lineages emerging in this patient. Six environmental 
1023 isolates from the room of patient 75 are also shown (light pink). Bootstrap values (\%) from 1000

1024 replicates have been displayed for major branches in the tree. 


\section{Figure 1 (on next page)}

Workflow summary diagram of methods used in this study.

Microbiology methods (orange), bioinformatic methods (blue), and epidemiological methods (green) used to generate data for analysis and reporting to public health authorities (purple) are shown. Results of combined prospective epidemiological and genomic analyses performed iteratively during the outbreak were reported to the Department of Health and Human Services in real-time. $\mathrm{CPE}=$ carbapenemase-producing Enterobacteriaceae; KPC = Klebsiella pneumoniae carbapenemase; MALDI-TOF = matrix-assisted laser desorption/ionisation time-of-flight; PCR = polymerase chain reaction; WGS = whole-genome sequencing; SNP = single nucleotide polymorphism; AMR = antimicrobial resistance; MLST = multi-locus sequence typing. 


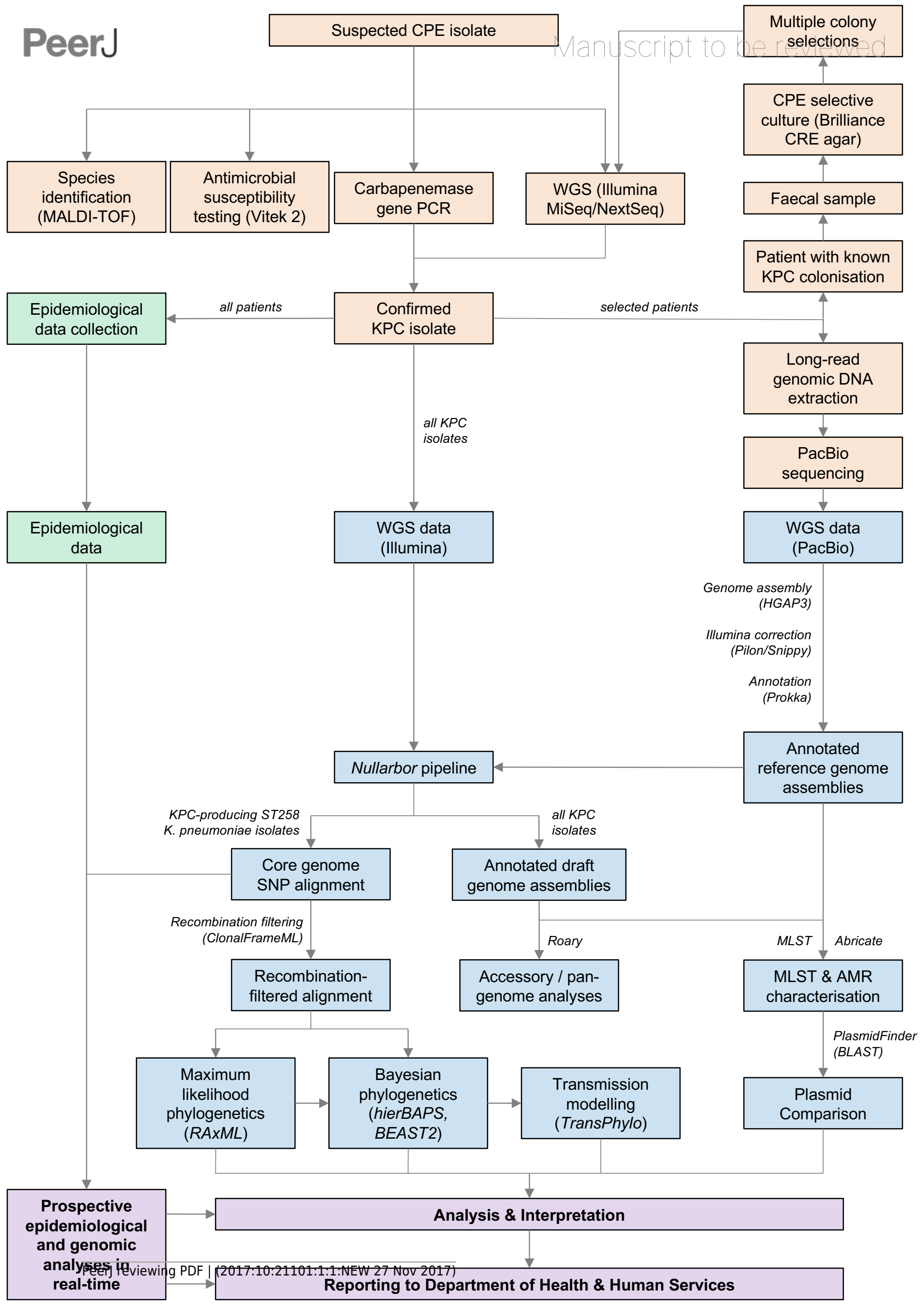


Figure 2 (on next page)

Incidence of new KPC-producing Enterobacteriaceae cases referred to MDU PHL, 20122015.

Blocks are coloured by the species and KPC allele of the referred isolates. Repeated detections of KPC-producing isolates from the same patient have been excluded. 


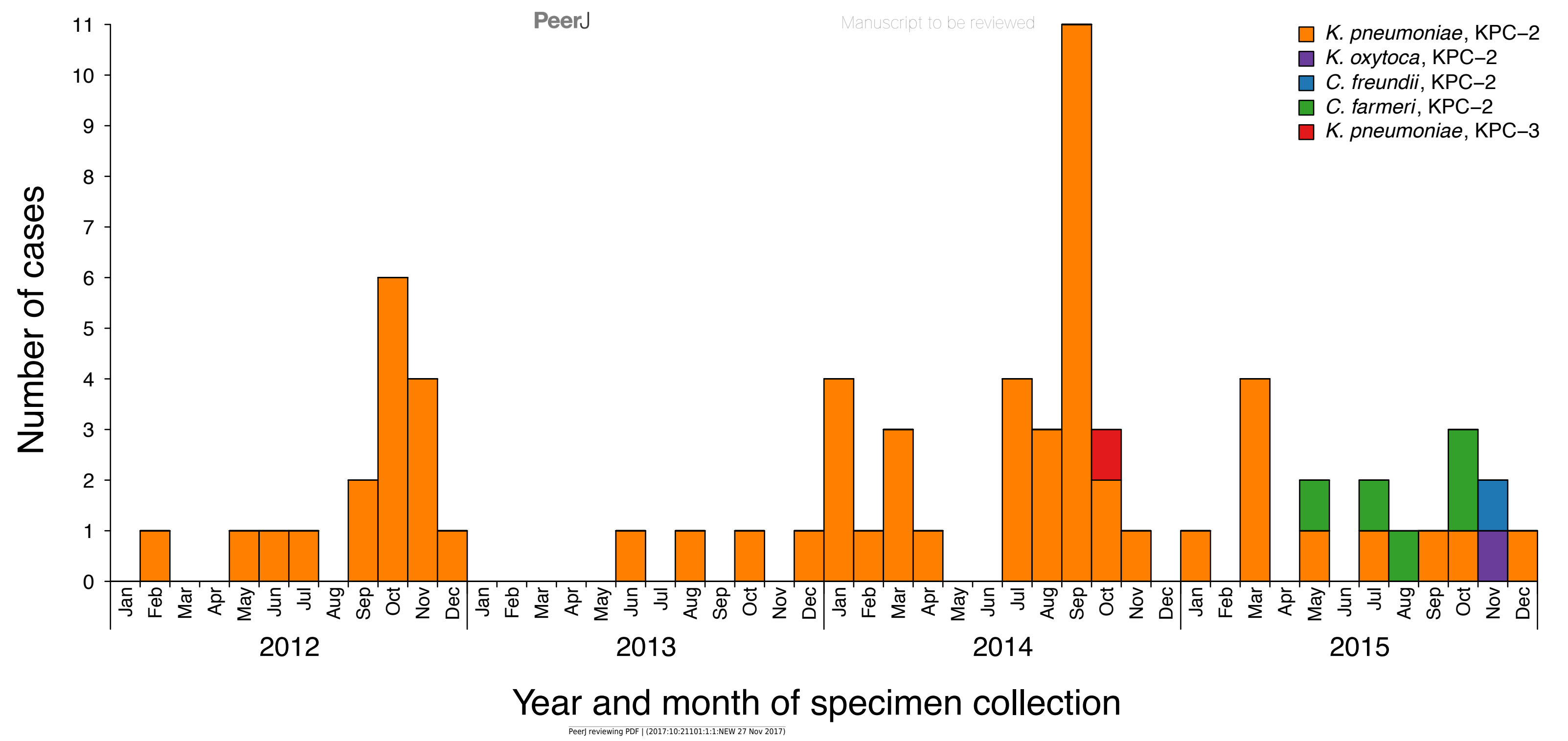




\section{Figure 3 (on next page)}

The initial maximum likelihood phylogenetic tree comprised three dominant clades.

The tree includes 29 ST258 K. pneumoniae isolates collected from 29 patients prior to June 2014, with external nodes coloured according to healthcare facility at time of sample collection. Recurrent isolates from each patient have been excluded. Multiple patients in Facility A (purple) and Facility F (orange) were colonised/infected with KPC-producing $K$. pneumoniae, corresponding to known previous outbreaks in those hospitals. Support values (\%) from 1000 bootstrap replicates are shown for major branches. Major phylogenetic clades have been labelled cluster A (green shading), B (orange shading), and C (purple shading) in the order that the clades emerged, with the larger clade B comprising two subclades, B1 and B2. Corresponding clusters identified through Bayesian Analysis of the Population Structure (BAPS) are also shown. The tree was rooted using an outgroup isolate ( $K$. pneumoniae NJST258_1, GenBank accession: CP006923.1; not shown in the tree) from a different ST258 clade. 


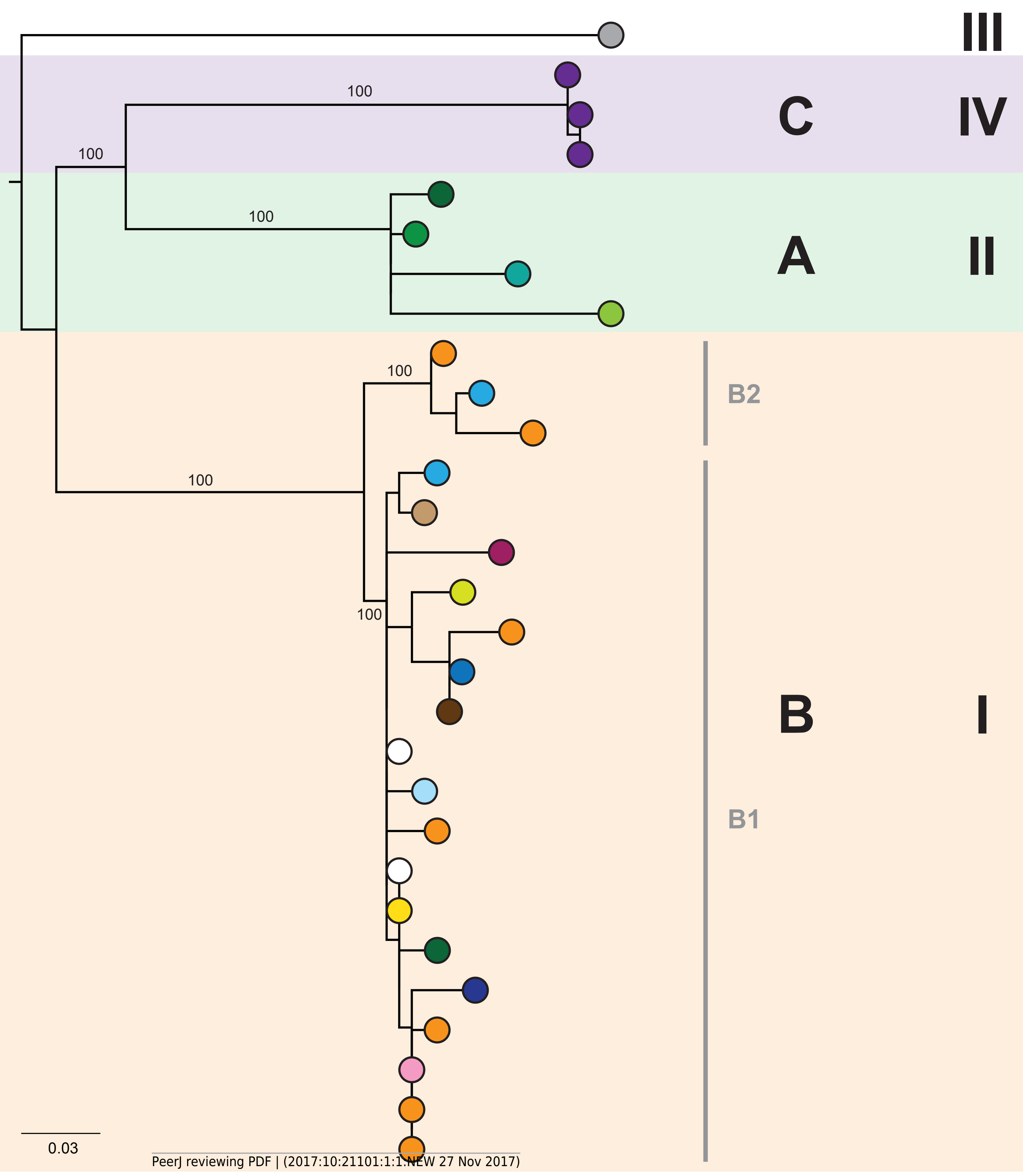




\section{Figure 4 (on next page)}

Epidemiological links based on overlapping patient admissions were unable to resolve where and when transmission was occurring for most isolates.

Links in the above network connect patients based on overlapping patient admissions in the same hospital ward at the same time (minimum of one day overlap). Links that occurred after detection of $b / a_{\text {KPC }}$ in both patients have been excluded. Nodes are numbered by patient, and coloured by healthcare facility (as in Figure 3 ) at the time of sample collection. The epidemiological network connecting patients 22, 23, 24 and 33 correlates with the closely related genomes in Cluster C (Figure 3). 
PeerJ

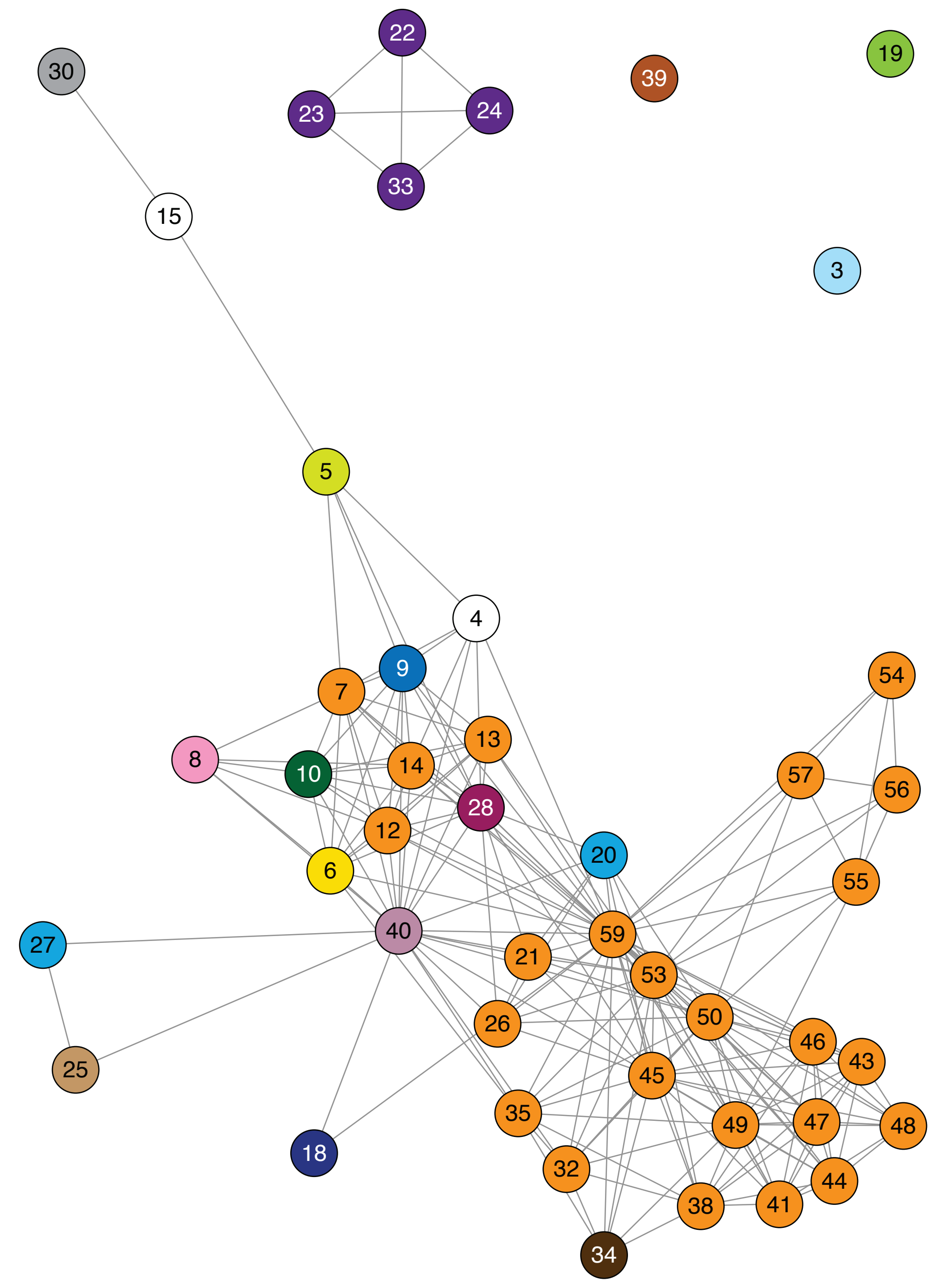

(29)

(16)

(31) 


\section{Figure $\mathbf{5}$ (on next page)}

Combined analysis, with genomic relationships between isolates overlaid upon epidemiological data, delineated multiple transmission networks.

A maximum likelihood phylogenetic tree is shown on the left, labelled with major genomic clusters and supporting branch bootstrap values (\%) from 1000 replicates for major branches. Nodes of the tree are coloured by healthcare facility at the time of sample collection as in Figures 3 and 4. Coloured horizontal bars on the right indicate healthcare facility admissions over time ( $x$ axis), with different colours representing different healthcare networks. Black diamonds (i) indicate first detection of KPC for each patient. 
PeerJ

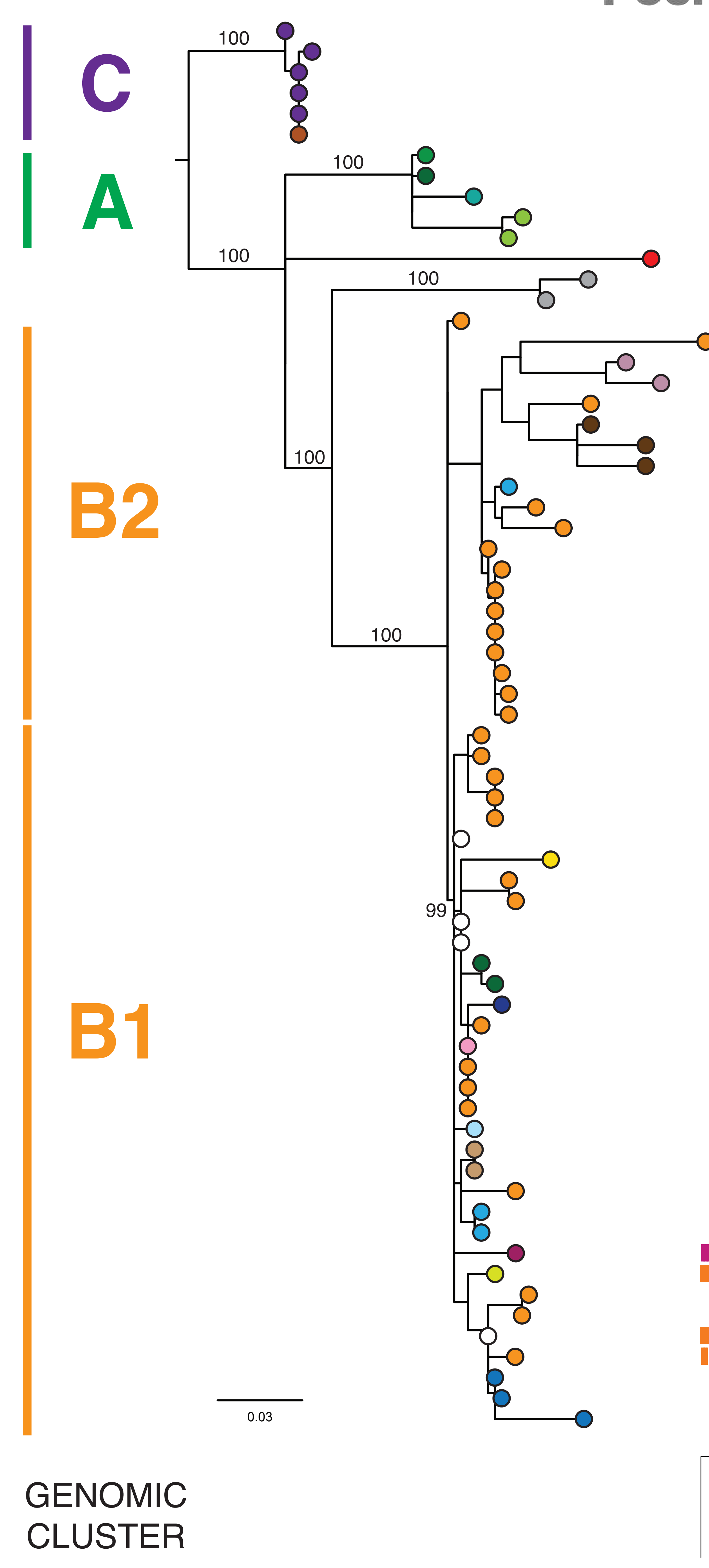

Manuscript to be reviewed

I

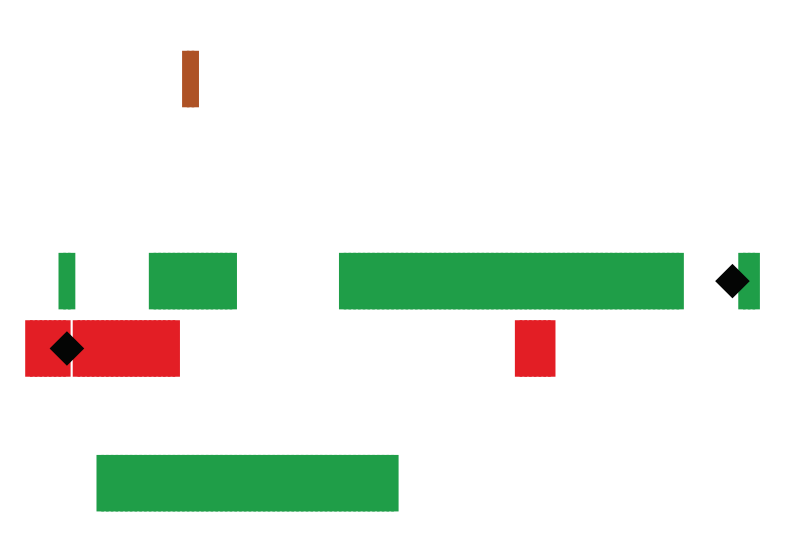

1

1 trent
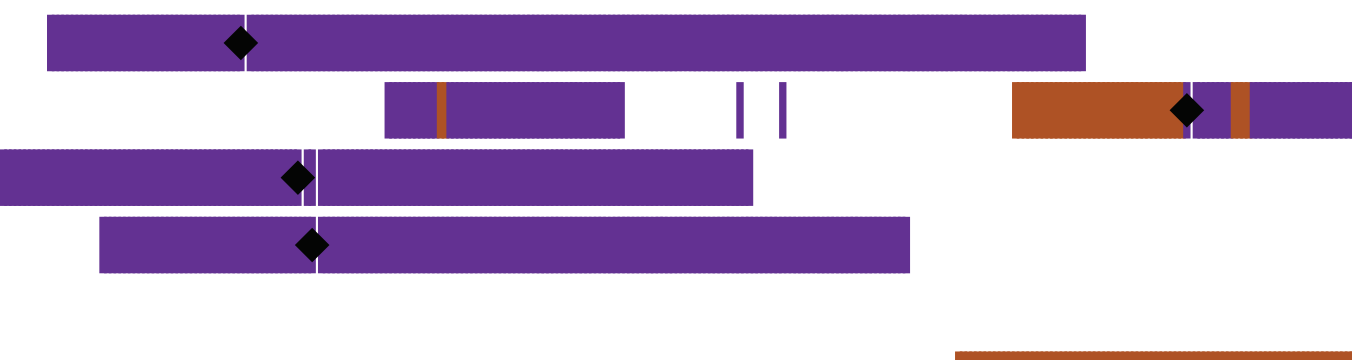

I |
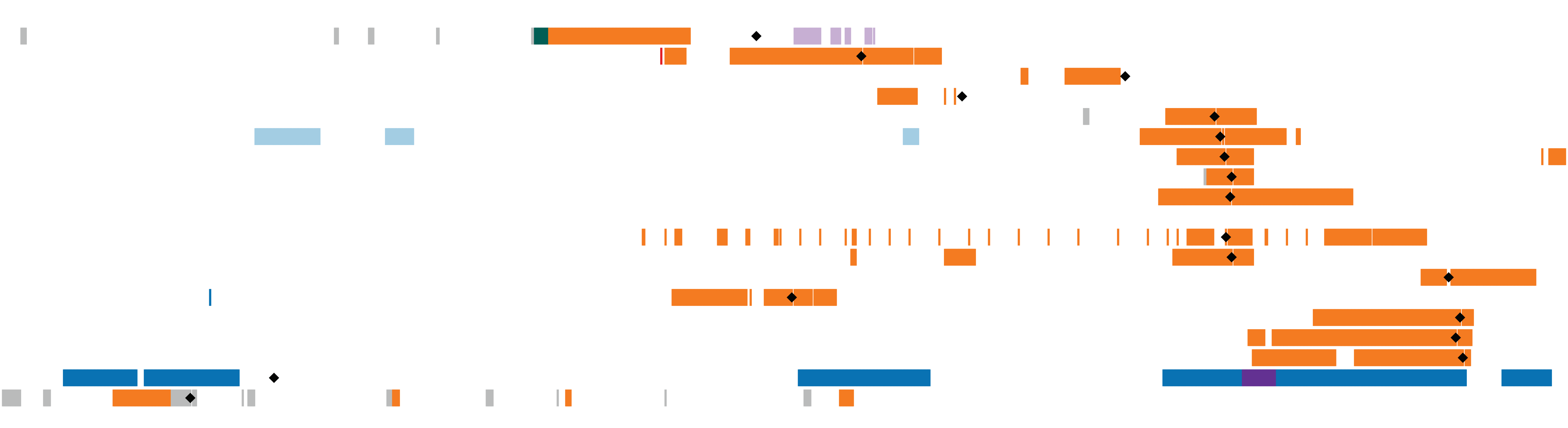
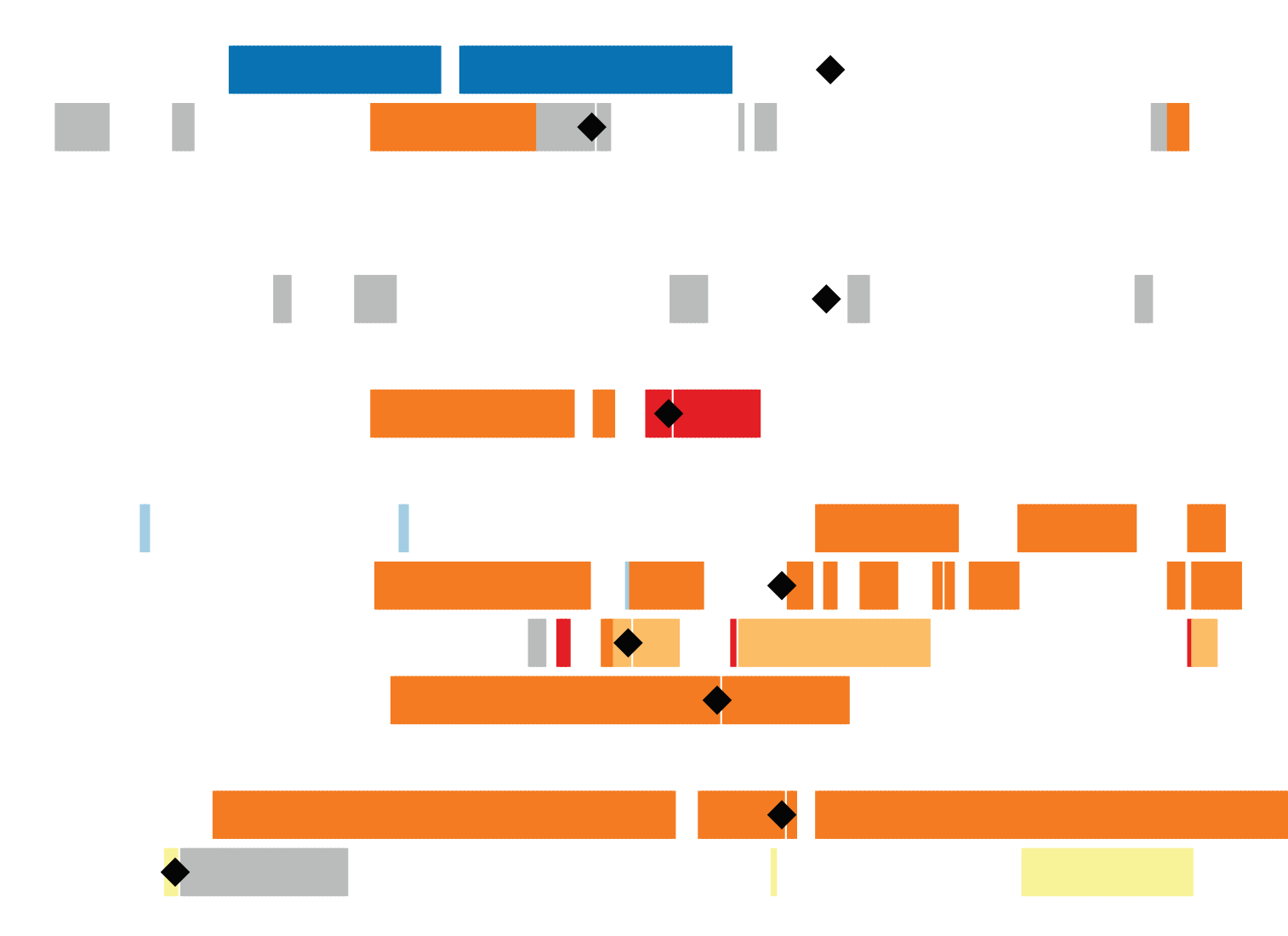

\section{-}

|

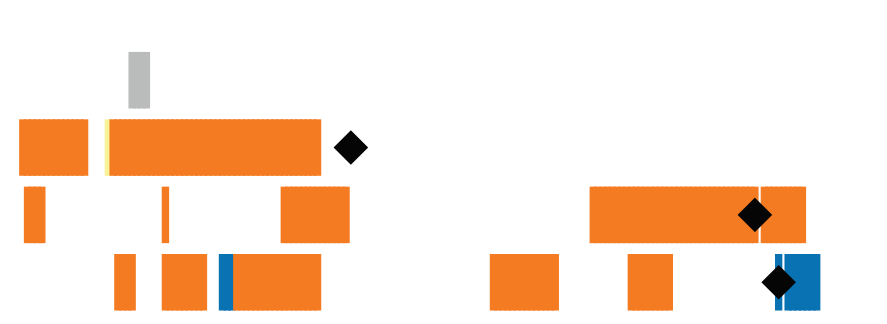

$\stackrel{\text { ब }}{2}$

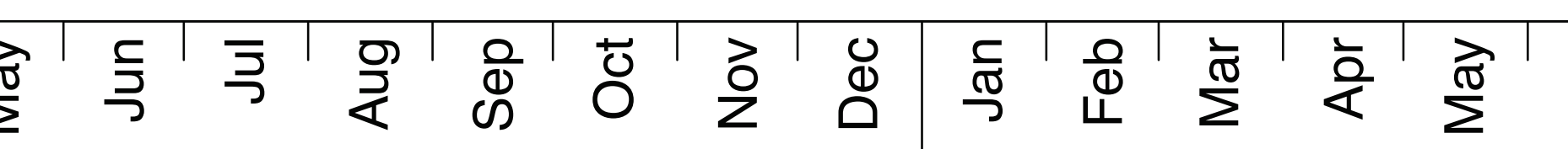
2012
| | | |

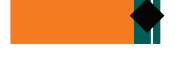

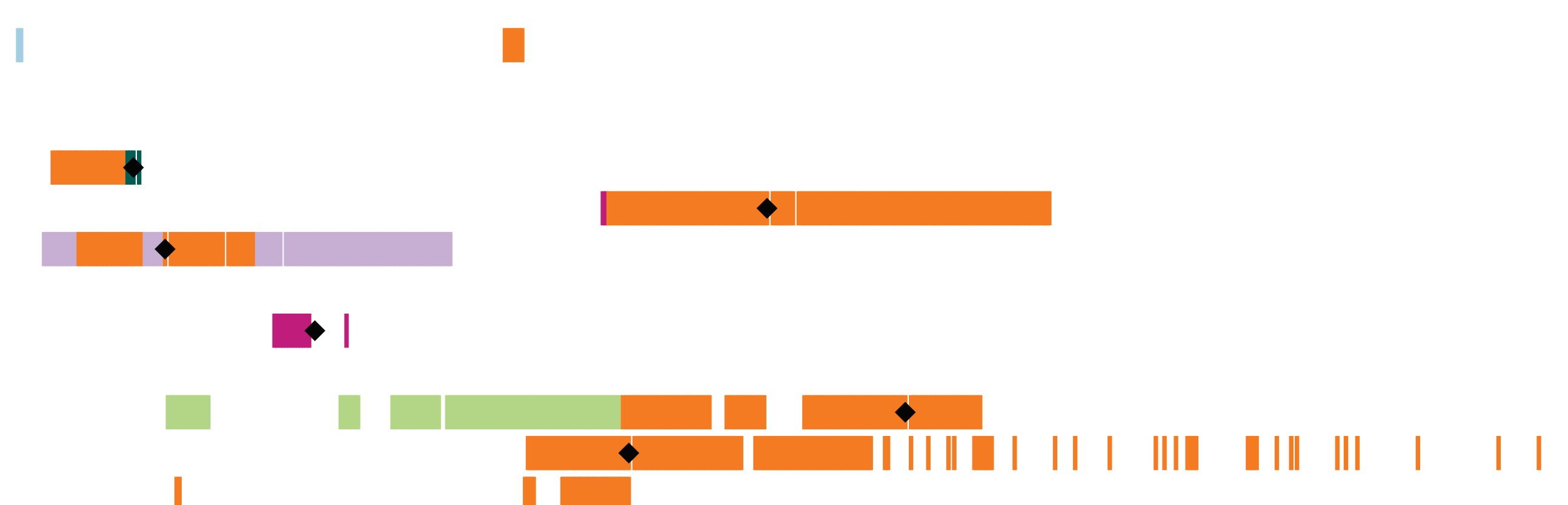

I 1

II | | |

| [ |
2014

\section{Year and month}




\section{Figure 6 (on next page)}

Combined analysis of genomic and epidemiological data of the cluster $\mathrm{C}$ network identified secondary transmission.

Example case study: Patients 22 and 23 both reported overseas hospitalisation in the 12 months prior to first detection of KPC - patient 22 in Vietnam for spinal surgery following a motor vehicle accident, and patient 23 in Greece for stem cell therapy. Both patients had undergone rectal screening on admission to Ward 1 in Facility A, with patient 22 being placed in intensive contact precautions for the duration of his hospital admissions after isolating another multidrug-resistant organism, though was required to use shared bathroom facilities with patients in the adjacent room. Having required treatment with meropenem for both hospital-acquired pneumonia and a surgical wound infection, patient 22 was later diagnosed with a KPC-producing $K$. pneumoniae indwelling catheter-associated urinary infection in January 2014. Twelve days later, patient 23 was subsequently found to have a polymicrobial sacral wound infection, with cultures including KPC-producing $K$. pneumoniae from sacral tissue. In response to this, all patients on the ward who had been admitted to the same room and/or shared bathroom facilities with patients 22 and 23 were screened, with the subsequent identification of patient 24. Alerts were placed on the records of patients meeting the criteria who had been previously discharged. Environmental screening of the rooms and bathrooms was conducted, with no KPC-producing organisms identified, and extended bleach cleaning with changes of curtains, chairs and other furnishings was conducted for the entire ward. Patient 33 was also admitted to Ward 1 in Facility A in February 2014, subsequent to the identification of KPC-2 in patients 22, 23 and 24. This patient was not screened as he had not been admitted to the same room, nor had he shared a bathroom with the identified cases. He also reported no recent history of overseas travel. However, he was identified in July 2014 through routine screening at Facility A following transfer from Ward 3, Facility U, located $25 \mathrm{~km}$ away. A KPC-producing isolate from Patient 39 was identified in September

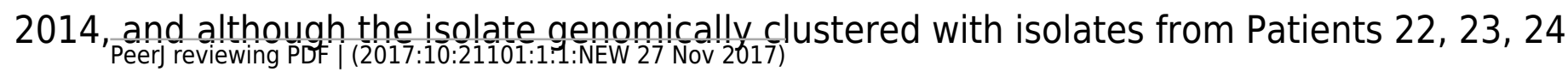


and 33 identified at Facility A, she had no previous presentations to that healthcare facility. However, immediately prior to identification of KPC, she had also been in Facility $U$ on Ward 3, though she was admitted there 13 days after Patient 33's discharge. Given this was the only plausible epidemiological link to the other cluster $\mathrm{C}$ patients, secondary transmission was presumed to have occurred in Ward 3, Facility $U$. 


\section{PATIENT}
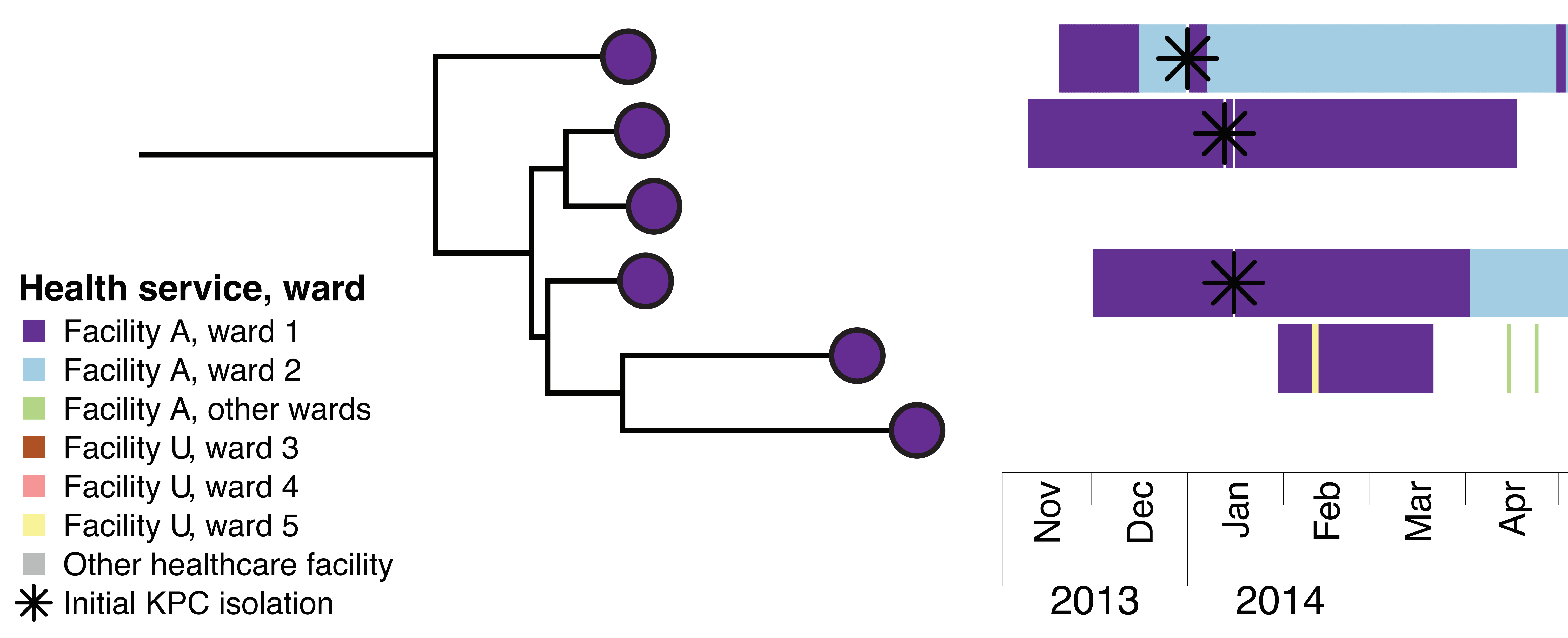

22

23

23

24

33 
Figure 7 (on next page)

Bayesian evolutionary analysis indicates each of the phylogenetic clades corresponding to the genomic clusters emerged prior to the detection of KPC in Victoria.

A maximum clade credibility timed phylogeny from Bayesian evolutionary analysis of local CC258 K. pneumoniae isolate genomes is shown, with median node heights displayed. The thin red bars indicate $95 \%$ highest posterior density (HPD) intervals for the most recent common ancestor (MRCA) for major clades and defined genomic clusters (indicated on the right). The shaded grey region indicates the recent period when KPC isolates were detected in Victoria. 


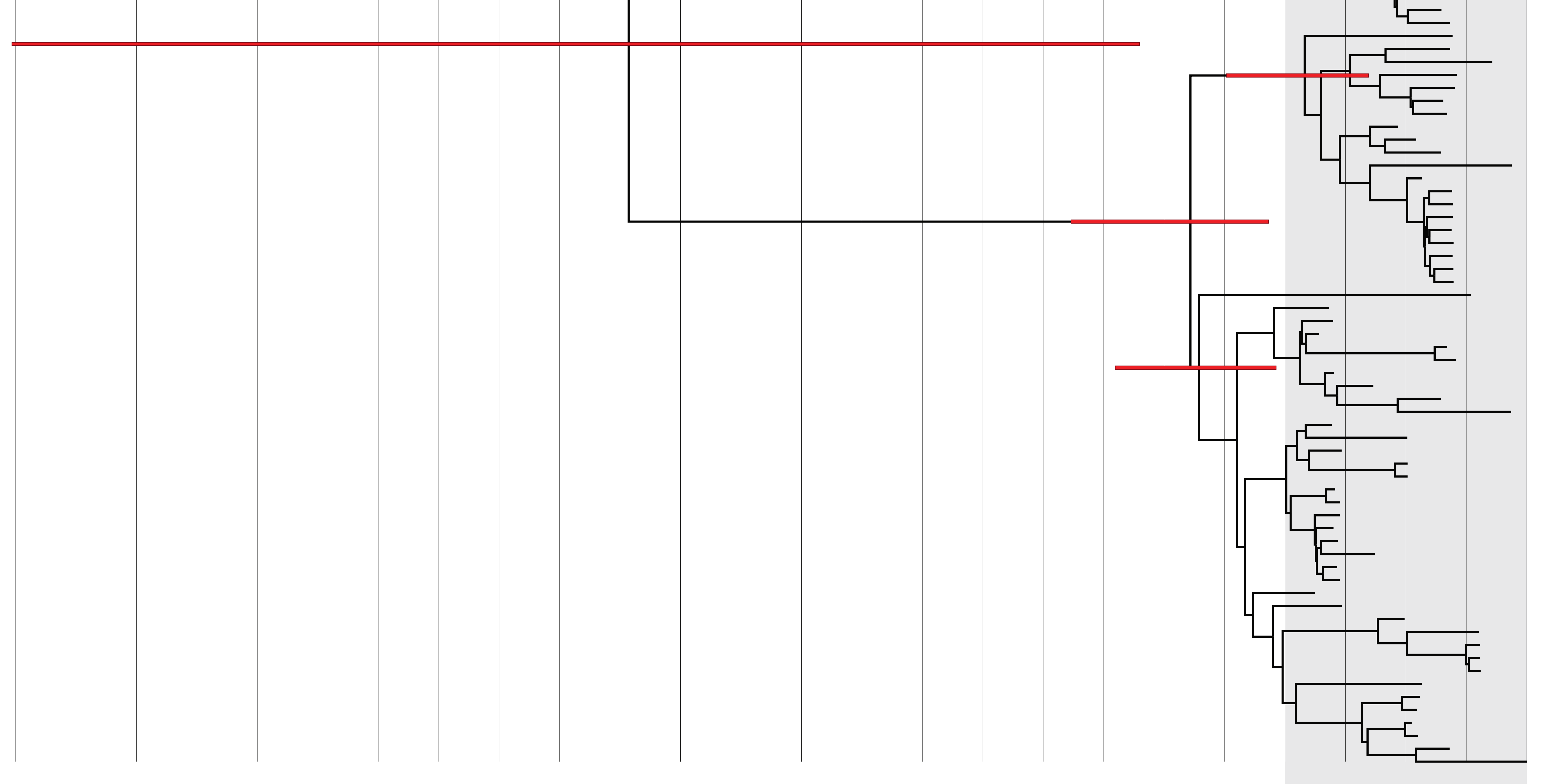

B2 
Figure 8 (on next page)

An inferred transmission tree shows that undetected colonisation was significant in propagating the outbreak.

Solid nodes represent the posterior mean time of KPC acquisition by individuals and are coloured by the corresponding genomic cluster, with empty circles representing inferred unsampled individuals contributing to the transmission tree. Braches are shaded by number of missing links in the transmission tree, with lighter branches representing increasing numbers of missing links implicated. 


\section{PeerJ}

\section{Manuscript to be reviewed}

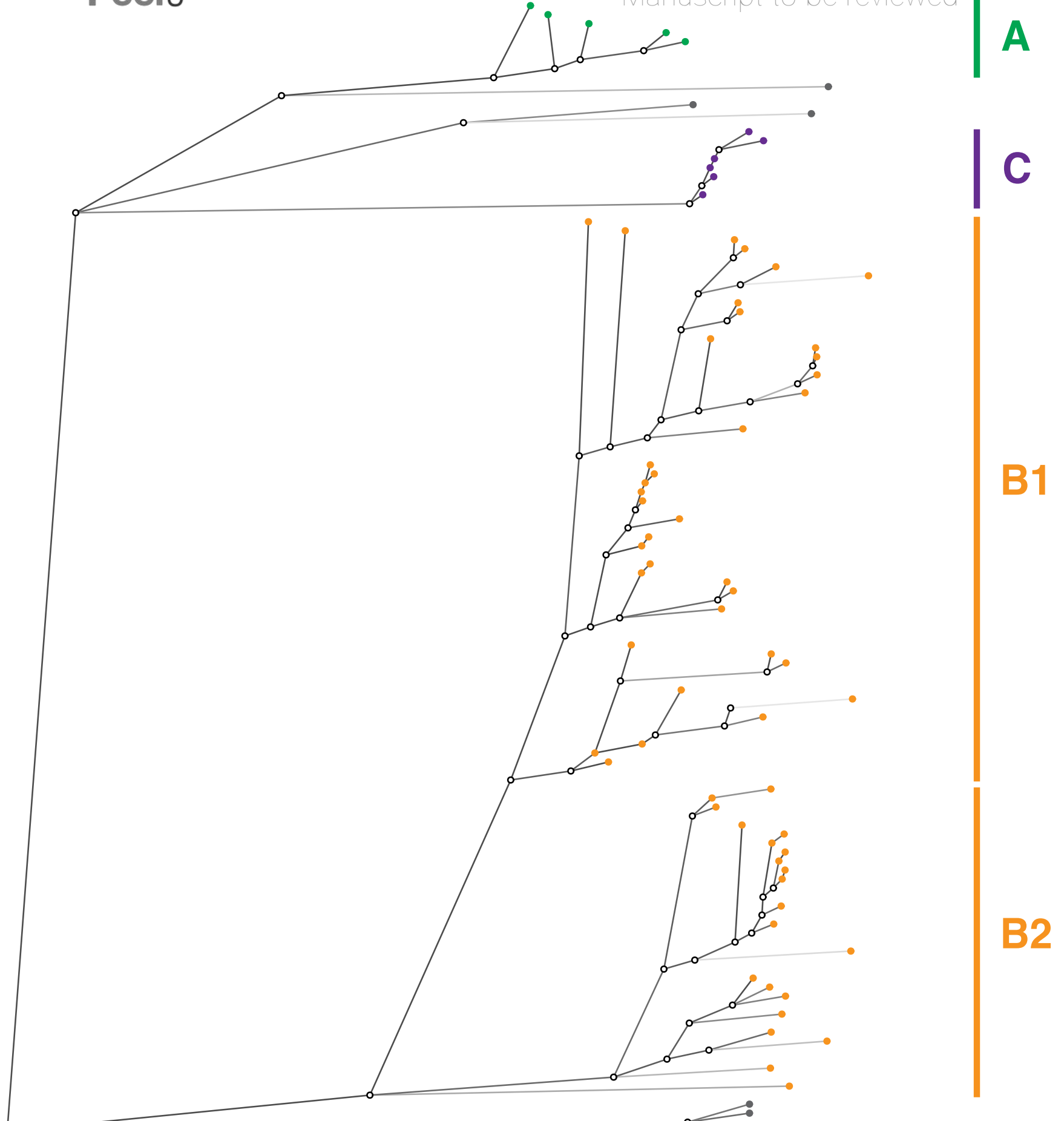

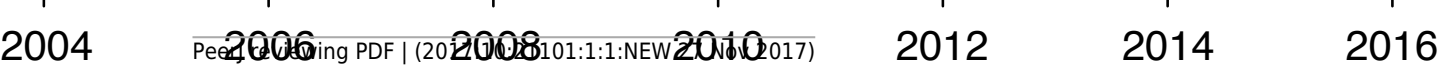


Figure 9 (on next page)

KPC plasmids from the $C$. farmeri isolates were almost identical to KPC plasmids from $K$. pneumoniae outbreak isolates, despite differing replication proteins.

BLAST comparison between an IncFIB (pQIL-like) plasmid genome from a ST258 Klebsiella pneumoniae isolate, AUSMDU00008079 (above), and an IncR plasmid genome from a Citrobacter farmeri isolate, AUSMDU00008141 (below), from the outbreak. The grey shading indicates corresponding DNA regions of high nucleotide identity transcribed in opposing directions, with the Tn4401 transposon harbouring $b / a_{\mathrm{KPC}-2}$ highlighted in red. The plasmid genomes have been orientated to their respective replicons and downstream plasmid partitioning genes, parA and parB. 


\section{IncFIB(pQIL)

parA

parB

$\operatorname{Tn} 4401$

repA

Type I

restriction

modification

system

repB $B$ (1) parA parB

transposase

$\operatorname{Tn} 4401$

IncR

Citrobacter farmeri 
Figure 10 (on next page)

Genomes of isolates from the same host group together in the phylogeny.

Maximum likelihood tree of the study isolates with additional isolates obtained up to 1 November 2016 included. Multiple isolates from the same patient have been coloured by patient, with the accompanying graph indicating the collection dates for the corresponding isolates. Thirty-two isolates from six clinical samples obtained from patient 70 (blue) over 8 months have been highlighted to illustrate the within-host lineages emerging in this patient. Six environmental isolates from the room of patient 75 are also shown (light pink). Bootstrap values (\%) from 1000 replicates have been displayed for major branches in the tree. 


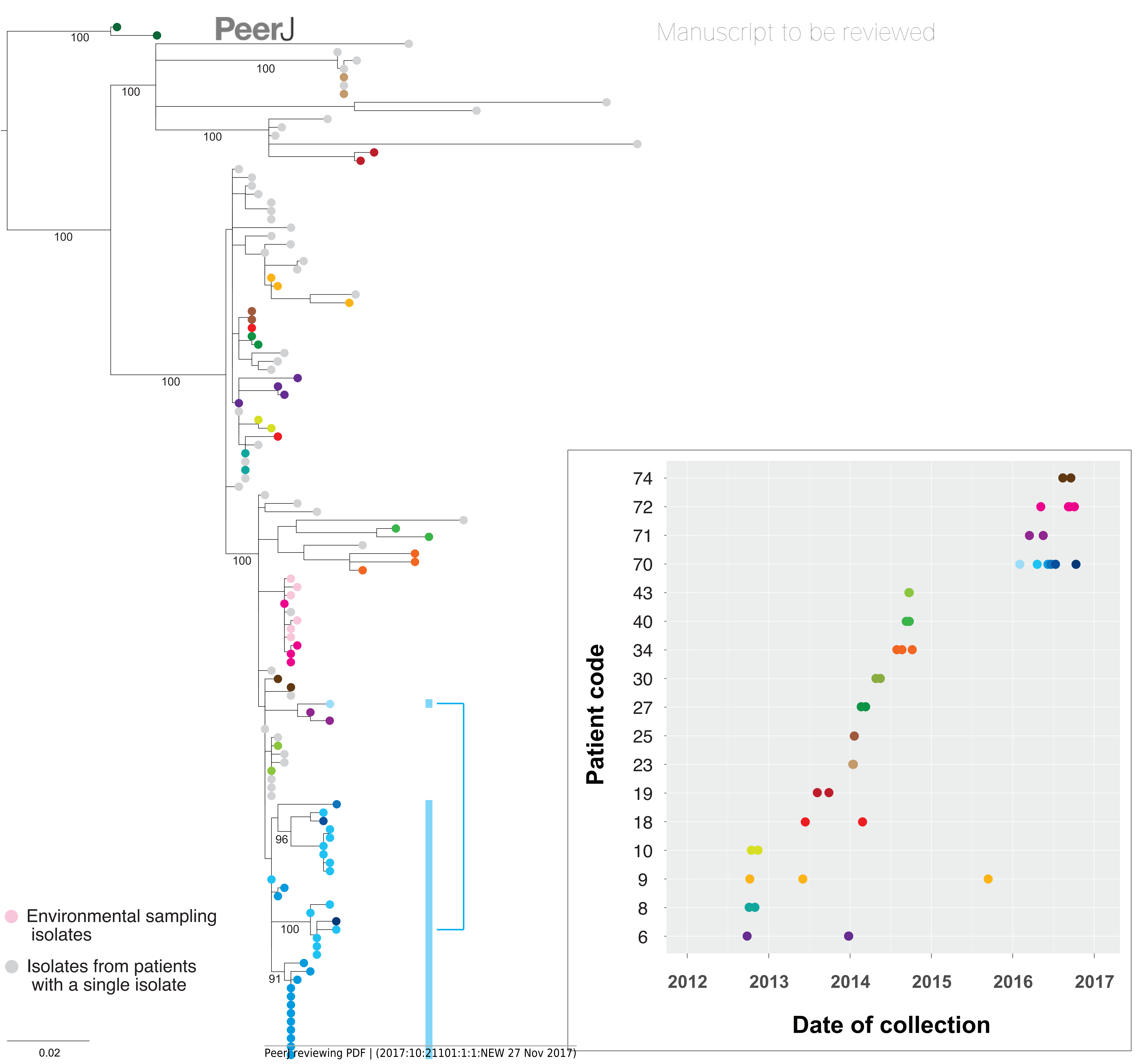

